\title{
SPECTRAL THEORY OF FIRST-ORDER SYSTEMS: FROM CRYSTALS TO DIRAC OPERATORS
}

\author{
MATANIA BEN-ARTZI AND TOMIO UMEDA
}

AbStract. Let

$$
L_{0}=\sum_{j=1}^{n} M_{j}^{0} D_{j}+M_{0}^{0}, \quad D_{j}=\frac{1}{i} \frac{\partial}{\partial x_{j}}, \quad x \in \mathbb{R}^{n},
$$

be a constant coefficient first-order partial differential system, where the matrices $M_{j}^{0}$ are Hermitian. It is assumed that the homogeneous part is strongly propagative. In the nonhomegeneous case it is assumed that the operator is isotropic. The spectral theory of such systems and their potential perturbations is expounded, and a Limiting Absorption Principle is obtained up to thresholds. Special attention is given to a detailed study of the Dirac and Maxwell operators.

The estimates of the spectral derivative near the thresholds are based on detailed trace estimates on the slowness surfaces. Two applications of these estimates are presented:

- Global spacetime estimates of the associated evolution unitary groups, that are also commonly viewed as decay esimates. In particular the Dirac and Maxwell systems are explicitly treated.

- The finiteness of the eigenvalues (in the spectral gap) of the perturbed Dirac operator is studied, under suitable decay assumptions on the potential perturbation.

\section{I INTRODUCTION}

The equations of classical physics, governing acoustic, electromagnetic and elastic waves in anisotropic media, are given as first-order hyperbolic systems. Similarly, the Dirac equation of relativistic quantum electrodynamics is such a system. Already in the classical treatise of Courant and Hilbert the common features of these systems are brought to the fore [17, Chapter III, §2, Chapter VI, §3a].

Date: February 11, 2019.

2010 Mathematics Subject Classification. Primary 35Q41; Secondary 35Q61, 35Q40.

Key words and phrases. Dirac operator, Maxwell equations, first-order systems, strongly propagative systems, spectral derivative, spacetime estimates, spectrum, limiting absorption principle, thresholds, eigenvalues in gap, perturbation by potential.

This work was started at the Newton Institute, Cambridge UK, when the two authors were participants in the programme "Spectral Theory of Relativistic Operators" (Summer 2012), organized by M. Brown, M. J. Esteban, K. Schmidt and H. Siedentop. The authors thank the Newton Institute and the organizers for their support. The University of Hyogo (Himeji) and Ritsumeikan University supported M. Ben-Artzi in his visit (March-April 2017) and the Hebrew University supported T. Umeda in his visit (January 2018), when this paper was completed. Special thanks are due to Professor H. Isozaki of Ritsumeikan University for his support and Professor H. Tamura for his valuable suggestions and constant interest in this work. We thank Professor V. Mazya for bringing the reference [28] to our attention. T.Umeda was partially supported by the Japan Society for the Promotion of Science "Grant-in-Aid for Scientific Research" (C) No. 26400175. 
Motivated by this approach, emphasizing a unifying point of view, we study here basic spectral properties of a class of first-order self-adjoint operators, that includes the aforementioned systems, or, more explicitly, their spatial generators.

Thus, we consider operators acting on $\mathbb{C}^{K}$-valued functions in $\mathbb{R}^{n}, n \geq 2$, having the form

$$
L=\sum_{j=1}^{n} M_{j}^{0} D_{j}+M_{0}^{0}+V(x), D_{j}=\frac{1}{i} \frac{\partial}{\partial x_{j}}, \quad x \in \mathbb{R}^{n},
$$

where the matrices $M_{j}^{0}, 0 \leq j \leq n$, are Hermitian $K \times K$ constant matrices and the matrix $V(x)$ is Hermitian for every $x \in \mathbb{R}^{n}$. Thus $L$ is symmetric (with respect to the $\mathcal{L}^{2}\left(\mathbb{R}^{n}, \mathbb{C}^{K}\right)$ scalar product) on $C_{0}^{\infty}\left(\mathbb{R}^{n}, \mathbb{C}^{K}\right)$.

The obvious notation

$$
\mathcal{L}^{2}\left(\mathbb{R}^{n}, \mathbb{C}^{K}\right)=\mathcal{L}^{2}\left(\mathbb{R}^{n}\right) \bigotimes \mathbb{C}^{K}
$$

(resp. $\left.C_{0}^{\infty}\left(\mathbb{R}^{n}, \mathbb{C}^{K}\right)=C_{0}^{\infty}\left(\mathbb{R}^{n}\right) \otimes \mathbb{C}^{K}\right)$ has been used for the space of Lebesgue square-integrable (resp. smooth and compactly supported) $\mathbb{C}^{K}$-valued functions.

Our objectives in this paper are:

- Establish a "Limiting Absorption Principle" (LAP) for the special cases of the Dirac and Maxwell operators (Section 4) up to the thresholds, with sharp resolvent estimates.

- Generalize the LAP to "strongly propagative" or "isotropic operators" (Section 5).

- Extend the LAP to the case of potential perturbation (Section 6).

- Exploit the sharp resolvent estimates of the Dirac and Maxwell operators near the thresholds in the study of two applications:

- Obtain decay conditions on the potential perturbation of the Dirac operator which guarantees the finiteness of the isolated eigenvalues in the spectral gap (Subsubsection 6.2.1).

- Derive global spacetime estimates of the solutions in weighted $L^{2}$ spaces (Section 7).

- Extend the global spacetime estimates of the solutions in weighted $L^{2}$ spaces to the case of strongly propagative systems (Section 8).

Both the Dirac (Subsection 2.1) and the Maxwell (Subsection 2.2) systems are isotropic. Their prime significance in physics justifies the detailed study of their spectral structure, as is carried out in Section 4 .

The operator $L$ is viewed as a perturbation of a constant coefficient, symmetric operator

$$
L_{0}=\sum_{j=1}^{n} M_{j}^{0} D_{j}+M_{0}^{0}, \quad D_{j}=\frac{1}{i} \frac{\partial}{\partial x_{j}}, \quad x \in \mathbb{R}^{n} .
$$

The cases where $L_{0}$ is either strongly propagative (Definition 5.1) or isotropic (Definition 5.15) are studied in Section 5

It will be seen that the LAP is closely connected to the geometry of the level sets of the characteristic surfaces ("normal", by the terminology of [17]) in $\mathbb{R}^{n}$. In the physical context they are referred to as "Slowness Surfaces" [47, Section 4].

The presence of the non-zero matrix $M_{0}^{0}$ is motivated by the "massive" Dirac operator (Subsection 2.1). The basic operator $L_{0}$ is then nonhomogeneous, meaning that the associated eigenvalues are not homogeneous functions (see Section 5 for 
details) and as a result the geometry of the "slowness surfaces" is more complex. As far as we know, the Dirac operator is the only instance of a nonhomogeneous operator that has been treated in the literature. In this paper we study the nonhomogeneous operator in the general isotropic case.

A general (potential) perturbation theory is presented in Section 6 As an immediate consequence of the LAP for the perturbed operator, it follows that the spectrum is absolutely continuous, except possibly for a discrete sequence of embedded eigenvalues.

The general theory is then applied to the perturbed Dirac operator, as an important example. The threshold estimates for the perturbed Dirac operator enable us (Subsubsection 6.2.1) to give criteria for the finiteness of eigenvalues in the spectral gap.

Remark that the perturbed Maxwell system can also be reduced to the case of potential perturbation [42, Section 1.4], but we choose not to treat it here in detail, as the paper is already quite long. We refer to [11, 25] and references therein for the study of the perturbed Maxwell operator in terms of self-adjointness and absolute continuity of the spectrum.

In Section 7 we establish global spacetime estimates for the Dirac and Maxwell operators, and in Section 8 such estimates are derived for general (homogeneous) strongly propagative operators.

The literature concerning various aspects of the spectral and scattering theory of first-order systems, as well as asymptotic decay of their solutions, is very extensive, hence our reference list is far from being comprehensive. Certainly there are many papers that well deserve being included in our list and of which we are not aware. However, we have made an effort to include references to works directly related to this paper and dealing primarily with the LAP (in interior intervals of the spectrum or at thresholds). Most of these papers were concerned with the Dirac operator. We refer to [34, 36, 40, 48, and references therein for classical treatments of decay of solutions of first-order symmetric systems, and to [33] in the case of exterior domains.

The first proof of the LAP for the (massive) Dirac operator with short range potential perturbation was obtained by Yamada [50. It was subsequently proved (imposing various hypotheses on the perturbation) by several authors (see [3, 12, 13, 35, 51 for the massive case and 18, 37 for the zero mass case), as well as the recent papers [15, 22] (and references therein) for both the massive and zero mass cases. Their treatments rely on the fact (see Equation (2.8) that the square of this operator is the Schrödinger operator. A weak* form of the LAP, using the methodology of conjugate operators, was obtained in 14. The LAP (for the Dirac operator) up to the threshold was proved in 29. Recently their method has been extended in [15] in order to obtain a global LAP for the massless Dirac Operator in all dimensions. The zero modes and zero resonances of massless Dirac operators were studied in 37] and eigenfunctions at the threshold energies were studied in 38. We refer also to [8, 44] for the closely related "relativistic Schrödinger operator".

For the Maxwell equations in crystal optics the LAP was established in 42, Theorem 1.2].

For a uniformly propagative system (see Definition [5.3) of the form $E(x)^{-1} \sum_{j=1}^{n} M_{j}^{0} D_{j}$ the LAP was established in [41, 49], and also in [39]. For the more general strongly 
propagative systems it was proved in [46, Lemma 2.1]. See however Remark 5.14 concerning this paper. These works dealt with interior intervals of the spectrum, whereas here we obtain detailed estimates at the thresholds.

We are not aware of any work where the LAP for the nonhomogeneous operator $L_{0}$ (namely, $M_{0}^{0} \neq 0$ ) has been addressed, other than the massive Dirac operator.

We refer to Remark 6.19 concerning previous studies of the finiteness of eigenvalues in the spectral gap, in the case of the perturbed (massive) Dirac operator.

\section{THE CLASS OF UNPERTURBED OPERATORS-DIRAC AND MAXWELL SYSTEMS}

The coefficients $M_{0}^{0}, M_{1}^{0}, \ldots, M_{n}^{0}$ of the unperturbed operator $L_{0}$ (1.2) are constant Hermitian $K \times K$ matrices (over $\mathbb{C}$ ). The addition of $M_{0}^{0}$ will enable us in particular to include the massive Dirac operator in our treatment.

The (unitary) Fourier transform is defined by

$$
(\mathcal{F} u)(\xi)=\widehat{u}(\xi)=(2 \pi)^{-\frac{n}{2}} \int_{\mathbb{R}^{n}} u(x) e^{-i<\xi, x>\mathbb{R}^{n}} d x .
$$

The constant coefficient operators are transformed into multiplication operators (by symbols).

The homogeneous part of $L_{0}$ is assumed to be strongly propagative [48, according to Definition 5.1 below.

We shall address this general case in Section 5 . We start here with the two most famous physical examples of such operators, namely, the Dirac and Maxwell systems. As we shall see, both systems (including the nonhomogeneous Dirac system with mass) share the property of being isotropic (Definition 5.15 below).

2.1. EXAMPLE: THE FREE DIRAC OPERATOR. As a special case we consider the free Dirac operator. It is the self-adjoint operator $H_{m}$ in $\mathcal{L}^{2}\left(\mathbb{R}^{3}, \mathbb{C}^{4}\right)=$ $\mathcal{L}^{2}\left(\mathbb{R}^{3}\right) \otimes \mathbb{C}^{4}$ given by

$$
H_{m}=\alpha \cdot D+m \beta, m \geq 0
$$

where

$$
D=\frac{1}{i} \nabla_{x}, \quad x \in \mathbb{R}^{3},
$$

and $\alpha=\left(\alpha_{1}, \alpha_{2}, \alpha_{3}\right)$ is the triplet of $4 \times 4$ Dirac matrices

$$
\alpha_{j}=\left(\begin{array}{cc}
O_{2} & \sigma_{j} \\
\sigma_{j} & O_{2}
\end{array}\right), \quad j=1,2,3 .
$$

Here $\mathrm{O}_{2}$ is the $2 \times 2$ zero matrix and the $2 \times 2$ matrices $\sigma_{j}$ (Pauli matrices) are given by

$$
\sigma_{1}=\left(\begin{array}{cc}
0 & 1 \\
1 & 0
\end{array}\right), \quad \sigma_{2}=\left(\begin{array}{cc}
0 & -i \\
i & 0
\end{array}\right), \quad \sigma_{3}=\left(\begin{array}{cc}
1 & 0 \\
0 & -1
\end{array}\right)
$$

and

$$
\beta=\left(\begin{array}{cc}
I_{2} & O_{2} \\
O_{2} & -I_{2}
\end{array}\right), I_{2}=\left(\begin{array}{cc}
1 & 0 \\
0 & 1
\end{array}\right)
$$


The symbol $M_{m}(\xi)$ (corresponding to $M_{0}(\xi)$ above) is a $4 \times 4$ Hermitian matrix given by

$$
M_{m}(\xi)=\alpha \cdot \xi+m \beta .
$$

It is readily verified that we have the following equality of self-adjoint operators in $L^{2}\left(\mathbb{R}^{3}, \mathbb{C}^{4}\right)$,

$$
\left(H_{m}\right)^{2}=\left(-\Delta+m^{2}\right) \bigotimes I_{4} .
$$

Claim 2.1. The eigenvalues of $M_{m}(\xi)$ are given by $\lambda_{ \pm}(\xi)= \pm \sqrt{|\xi|^{2}+m^{2}}$, and are both of double multiplicity (except for $m=0$ and $\xi=0$ ).

It follows that the homogeneous operator $H_{0}$ is uniformly propagative (Definition 5.3).

For any $\xi \in \mathbb{R}^{3}$ (assuming either $\xi \neq 0$ or $m \neq 0$ ) there exists a unitary matrix $U_{m}(\xi)$ such that

$$
U_{m}(\xi)^{*} M_{m}(\xi) U_{m}(\xi)=\left(\begin{array}{cc}
\lambda_{+}(\xi) I_{2} & O_{2} \\
O_{2} & \lambda_{-}(\xi) I_{2}
\end{array}\right) .
$$

For $f \in \mathcal{L}^{2}\left(\mathbb{R}^{3}, \mathbb{C}^{4}\right)$ we define the transformation

$$
\left(\mathcal{G}_{m} f\right)(\xi)=U_{m}^{*}(\xi) \widehat{f}(\xi), \xi \in \mathbb{R}^{3} .
$$

Then

$$
\mathcal{G}_{m}: \mathcal{L}_{x}^{2}\left(\mathbb{R}^{3}, \mathbb{C}^{4}\right) \rightarrow \mathcal{L}_{\xi}^{2}\left(\mathbb{R}^{3}, \mathbb{C}^{4}\right)
$$

is unitary and diagonalizes $H_{m}$ in the sense that $\mathcal{G}_{m} H_{m} \mathcal{G}_{m}^{-1}$ in $\mathcal{L}_{\xi}^{2}\left(\mathbb{R}^{3}, \mathbb{C}^{4}\right)$ is given by the diagonal multiplication operator

$$
\left(\mathcal{G}_{m} H_{m} \mathcal{G}_{m}^{-1} \widehat{f}\right)(\xi)=\left(\begin{array}{cc}
\lambda_{+}(\xi) I_{2} & O_{2} \\
O_{2} & \lambda_{-}(\xi) I_{2}
\end{array}\right) \widehat{f}(\xi)
$$

In fact, in the physical literature this transformation is known as the FoldyWouthuysen-Tani transformation [26]. The transformation is explicitly presented (as can be easily verified) by [43, Section 1.4] and [2, Section 2.1]:

$$
\begin{gathered}
U_{m}(\xi)=\frac{1}{\sqrt{2} \sqrt{\lambda_{+}(\xi)^{2}+m \lambda_{+}(\xi)}}\left\{\left(\lambda_{+}(\xi)+m\right) I_{4}+\beta(\alpha \cdot \xi)\right\} \\
=\frac{1}{\sqrt{2} \sqrt{\lambda_{+}(\xi)^{2}+m \lambda_{+}(\xi)}}\left(\begin{array}{cccc}
\lambda_{+}(\xi)+m & 0 & -\xi_{3} & -\xi_{1}+i \xi_{2} \\
0 & \lambda_{+}(\xi)+m & -\xi_{1}-i \xi_{2} & \xi_{3} \\
\xi_{3} & \xi_{1}-i \xi_{2} & \lambda_{+}(\xi)+m & 0 \\
\xi_{1}+i \xi_{2} & -\xi_{3} & 0 & \lambda_{+}(\xi)+m
\end{array}\right)
\end{gathered}
$$

Clearly, the columns of this matrix are the eigenvectors of the symbol matrix $M_{m}(\xi)$.

We shall need this transformation when studying the spectral structure of the Dirac operator in Subsection 4.1 .

Remark 2.2. Another representation of (2.12) is given by (see [3])

$$
\begin{gathered}
U_{m}(\xi)=\exp \left\{-\beta(\alpha \cdot \xi) \theta_{m}(|\xi|)\right\}, \\
\theta_{m}(t)=(2 t)^{-1} \arctan \left(m^{-1} t\right), \quad m, t>0 .
\end{gathered}
$$


Observe that due to the double multiplicity of $\lambda_{ \pm}(\xi)$ the diagonalizing matrix $U_{m}(\xi)$ is not unique.

2.2. EXAMPLE: THE FREE MAXWELL OPERATOR. Consider a pair of three-dimensional vector functions $E$ (the electric field) and $B$ (the magnetic field). We shall denote by $\left(\begin{array}{l}E \\ B\end{array}\right)$ the six-component (column) vector that consists of the vertical arrangement of $E, B$.

The free (vacuum) Maxwell operator $L_{\text {maxwell }}$ is a $6 \times 6$ self-adjoint operator acting on the combined vector $\left(\begin{array}{l}E \\ B\end{array}\right)$ in $\mathcal{L}^{2}\left(\mathbb{R}^{3}, \mathbb{C}^{6}\right)=\mathcal{L}^{2}\left(\mathbb{R}^{3}\right) \otimes \mathbb{C}^{6}$,

$$
L_{\text {maxwell }}\left(\begin{array}{l}
E \\
B
\end{array}\right)=\frac{1}{i}\left(\begin{array}{cc}
O_{3} & -\operatorname{curl} \\
\text { curl } & O_{3}
\end{array}\right)\left(\begin{array}{l}
E \\
B
\end{array}\right)=\left(\begin{array}{cc}
O_{3} & -D \times \\
D \times & O_{3}
\end{array}\right)\left(\begin{array}{l}
E \\
B
\end{array}\right),
$$

where as above $D=\left(D_{1}, D_{2}, D_{3}\right)=\frac{1}{i} \nabla_{x}, \quad x \in \mathbb{R}^{3}$, and $O_{3}$ is the $3 \times 3$ zero matrix.

Remark 2.3. Recall that the electric (E) and magnetic (B) fields in vacuum are divergence-free fields, namely, there are no electric or magnetic charges. This restriction is imposed on the initial data and then the time-dependent equations ensure that the fields evolve as divergence-free fields.

However, here we take the point-of-view that $L_{\text {maxwell }}$ acts on the entire space $\mathcal{L}^{2}\left(\mathbb{R}^{3}, \mathbb{C}^{6}\right)$.

For the Maxwell operator $L_{\text {maxwell }}$ in (2.14), the matrix symbol $M_{\text {maxwell }}(\xi)$ is readily seen to be of the form

$$
M_{\text {maxwell }}(\xi)=\left(\begin{array}{cc}
O_{3} & -\gamma(\xi) \\
\gamma(\xi) & O_{3}
\end{array}\right),
$$

where, for $\xi=\left(\xi_{1}, \xi_{2}, \xi_{3}\right)$,

$$
\gamma(\xi)=\left(\begin{array}{ccc}
0 & -\xi_{3} & \xi_{2} \\
\xi_{3} & 0 & -\xi_{1} \\
-\xi_{2} & \xi_{1} & 0
\end{array}\right) .
$$

The eigenvalues of $M_{\text {maxwell }}(\xi), \xi \neq 0$, are

$$
\lambda_{+}(\xi)=|\xi|>\lambda_{0}(\xi)=0>\lambda_{-}(\xi)=-|\xi|,
$$

and the multiplicity of each of them is two. Since $\gamma(\xi) \xi=0$, it follows that the kernel of $M_{\text {maxwell }}(\xi)$ is two-dimensional, with basis vectors

$$
\left(\begin{array}{c}
0 \\
0 \\
0 \\
\xi_{1} \\
\xi_{2} \\
\xi_{3}
\end{array}\right), \quad\left(\begin{array}{c}
\xi_{1} \\
\xi_{2} \\
\xi_{3} \\
0 \\
0 \\
0
\end{array}\right)
$$

Thus the kernel is two-dimensional, independently of $\xi \in \mathbb{R}^{3} \backslash\{0\}$. It follows that this operator is uniformly propagative (Definition 5.3 below).

In order to construct the full system of eigenvectors of $M_{\text {maxwell }}(\xi)$ we introduce (for any $\xi \neq 0$ ) the following vectors in $\mathbb{C}^{3}$ : 


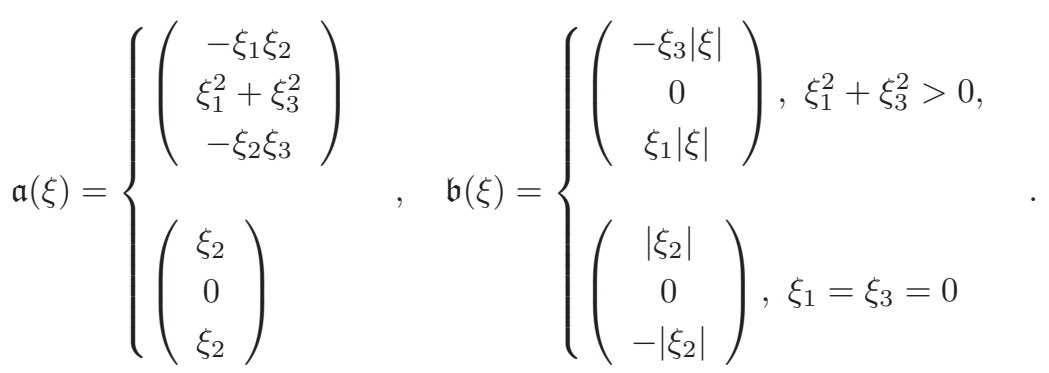

It is readily seen that the vectors $\mathfrak{a}(\xi), \mathfrak{b}(\xi), \xi$ are mutually orthogonal in $\mathbb{C}^{3}$. They also form a right-hand orthogonal system in $\mathbb{R}^{3}$, and satisfy the relations

$$
\gamma(\xi) \mathfrak{a}(\xi)=|\xi| \mathfrak{b}(\xi), \quad \gamma(\xi) \mathfrak{b}(\xi)=-|\xi| \mathfrak{a}(\xi) .
$$

In the following corollary, we write a (column) vector in $\mathbb{C}^{6}$ as a column with two (column) $\mathbb{C}^{3}$ components.

Corollary 2.4. Consider the following three pairs of vectors in $\mathbb{C}^{6}$

$$
\begin{gathered}
\Upsilon_{+}=\left\{\frac{1}{\sqrt{|\mathfrak{a}(\xi)|^{2}+|\mathfrak{b}(\xi)|^{2}}}\left(\begin{array}{c}
\mathfrak{a}(\xi) \\
\mathfrak{b}(\xi)
\end{array}\right), \frac{1}{\sqrt{|\mathfrak{a}(\xi)|^{2}+|\mathfrak{b}(\xi)|^{2}}}\left(\begin{array}{c}
-\mathfrak{b}(\xi) \\
\mathfrak{a}(\xi)
\end{array}\right)\right\}, \\
\Upsilon_{0}=\left\{\frac{1}{|\xi|}\left(\begin{array}{c}
0 \\
\xi
\end{array}\right), \frac{1}{|\xi|}\left(\begin{array}{c}
\xi \\
0
\end{array}\right)\right\} \\
\Upsilon_{-}=\left\{\frac{1}{\sqrt{|\mathfrak{a}(\xi)|^{2}+|\mathfrak{b}(\xi)|^{2}}}\left(\begin{array}{c}
-\mathfrak{a}(\xi) \\
\mathfrak{b}(\xi)
\end{array}\right), \frac{1}{\sqrt{|\mathfrak{a}(\xi)|^{2}+|\mathfrak{b}(\xi)|^{2}}}\left(\begin{array}{c}
\mathfrak{b}(\xi) \\
\mathfrak{a}(\xi)
\end{array}\right)\right\} .
\end{gathered}
$$

Then the set of six vectors $\Upsilon_{+} \cup \Upsilon_{0} \cup \Upsilon_{-} \subseteq \mathbb{C}^{6}$ constitutes an orthonormal basis of eigenvectors of $M_{\text {maxwell }}(\xi)$.

The pairs $\Upsilon_{ \pm}$(resp. $\Upsilon_{0}$ ) are eigenvectors associated with $\lambda_{ \pm}(\xi)$ (resp. $\lambda_{0}(\xi)$ ).

Remark 2.5. The double multiplicities of the eigenvalues implies that the basis vectors in each subspace are not uniquely determined. This explains the apparent asymmetry (with respect to $\left(\xi_{1}, \xi_{2}, \xi_{3}\right)$ ) of the eigenvectors in each of the sets $\Upsilon_{ \pm}, \Upsilon_{0}$. The operator (and its symbol $M_{\text {maxwell }}(\xi)$ ) is clearly symmetric with respect to orthogonal rotations in $\mathbb{R}^{3}$.

Remark 2.6. (propagation modes) Let $\left(\begin{array}{l}E_{0}(x) \\ B_{0}(x)\end{array}\right)$ be a vector function (with values in $\left.\mathbb{C}^{6}\right)$ whose Fourier transform $\left(\widehat{\bar{B}_{0}}(\xi)\right) \in \operatorname{span}\left\{\Upsilon_{ \pm}\right\}$, for every $0 \neq \xi \in \mathbb{R}^{3}$. In other words, $\left(\begin{array}{c}\widehat{E_{0}}(\xi) \\ \bar{B}_{0}(\xi)\end{array}\right)$ is orthogonal to $\operatorname{ker}\left(M_{\text {maxwell }}(\xi)\right)$ for all $\xi \neq 0$. Then in particular

$$
<\widehat{E_{0}}(\xi), \xi>_{\mathbb{C}^{3}}=0, \quad<\widehat{B_{0}}(\xi), \xi>_{\mathbb{C}^{3}}=0, \quad \xi \in \mathbb{R}^{3} .
$$

The propagation of the initial data $\left(\begin{array}{l}E_{0}(x) \\ B_{0}(x)\end{array}\right)$ by the time-dependent Maxwell system yields the solution $\left(\begin{array}{l}E(x, t) \\ B(x, t)\end{array}\right)$. Both $E(x, t)$ and $B(x, t)$ are superpositions of the plane waves $e^{i(<x, \xi> \pm t|\xi|)} \mathfrak{a}(\xi)$ and $e^{i(<x, \xi> \pm t|\xi|)} \mathfrak{b}(\xi)$, each of which is transverse (in fact orthogonal) to the propagation directions $\xi$ or $-\xi$. 
The terminology of "TE-modes" (resp. "TM-modes"), introduced by Lord Rayleigh in 1897, is used to characterize such fields.

The behavior of these waves for large time is further studied below in Subsection 7.2.

The diagonalization procedure of the Dirac operator (see (2.11) can now be repeated, with suitable modifications, for the Maxwell operator as follows.

We define a continuous map

$$
\xi \hookrightarrow V_{0}(\xi), \quad \xi \in \mathbb{R}^{3} \backslash\{0\},
$$

where $V_{0}(\xi)$ is unitary for all $\xi \in \mathbb{R}^{3} \backslash\{0\}$, so that

$$
V_{0}(\xi)^{*} M_{\text {maxwell }}(\xi) V_{0}(\xi)=\left(\begin{array}{ccc}
\lambda_{+}(\xi) I_{2} & O_{2} & O_{2} \\
O_{2} & O_{2} & O_{2} \\
O_{2} & O_{2} & \lambda_{-}(\xi) I_{2}
\end{array}\right) .
$$

We can clearly assume that $V_{0}(\xi)$ is homogeneous of order zero; $V_{0}(\beta \xi)=V_{0}(\xi), \beta>$ 0 .

Remark 2.7. Continuing Remark 2.5, the fact that the eigenvalues are "separated" and are of constant multiplicity implies [30. Chapter II.1.4] that the projections on the eigenspaces are continuous (and indeed real-analytic), hence this is true also for the map

$$
\xi \hookrightarrow V_{0}(\xi), \quad \xi \neq 0 .
$$

Remark that the choice of the unitary matrix is not unique. In particular, taking the matrix whose columns are the six vectors $\Upsilon_{+} \cup \Upsilon_{0} \cup \Upsilon_{-}$, yields a unitary matrix that diagonalizes $M_{\text {maxwell }}(\xi)$, but is not continuous (as a function of $\xi$ ).

For $f \in \mathcal{L}^{2}\left(\mathbb{R}^{3}, \mathbb{C}^{6}\right)$ we define the transformation

$$
\left(\mathcal{T}_{0} f\right)(\xi)=V_{0}^{*}(\xi) \widehat{f}(\xi), \xi \in \mathbb{R}^{3} \backslash\{0\} .
$$

Then

$$
\mathcal{T}_{0}: \mathcal{L}_{x}^{2}\left(\mathbb{R}^{3}, \mathbb{C}^{6}\right) \rightarrow \mathcal{L}_{\xi}^{2}\left(\mathbb{R}^{3}, \mathbb{C}^{6}\right),
$$

is unitary and diagonalizes $L_{\text {maxwell }}$ in the sense that

$$
\left(\mathcal{T}_{0} L_{\text {maxwell }} \mathcal{T}_{0}^{-1} \widehat{f}\right)(\xi)=\left(\begin{array}{ccc}
\lambda_{+}(\xi) I_{2} & O_{2} & O_{2} \\
O_{2} & O_{2} & O_{2} \\
O_{2} & O_{2} & \lambda_{-}(\xi) I_{2}
\end{array}\right) \widehat{f}(\xi), \xi \in \mathbb{R}^{3},
$$

is a multiplication by a diagonal matrix.

Remark 2.8. Note that the diagonalization equation applies only to $f$ in the domain of $L_{\text {maxwell. }}$ This will be discussed below, in Subsection 4.2.

\section{WEIGHTED SOBOLEV SPACES and BASIC NOTATION}

Here we introduce the weighted Sobolev spaces which play a basic role in our treatment. We shall only need these spaces in the framework of $\mathcal{L}^{2}$.

For $s \in \mathbb{R}$ and $p$ a nonnegative integer we define:

$$
\mathcal{L}^{2, s}\left(\mathbb{R}^{n}\right):=\left\{u(x) \quad / \quad\|u\|_{0, s}^{2}=\int_{\mathbb{R}^{n}}\left(1+|x|^{2}\right)^{s}|u(x)|^{2} d x<\infty\right\},
$$




$$
\mathcal{H}^{p, s}\left(\mathbb{R}^{n}\right):=\left\{u(x) \quad / \quad \nabla^{\alpha} u \in \mathcal{L}^{2, s}, \quad|\alpha| \leq p, \quad\|u\|_{p, s}^{2}=\sum_{|\alpha| \leq p}\left\|\nabla^{\alpha} u\right\|_{0, s}^{2}\right\} .
$$

The scalar product in $\mathcal{L}^{2, s}\left(\mathbb{R}^{n}\right)$ is

$$
(u, v)_{0, s}=\int_{\mathbb{R}^{n}}\left(1+|x|^{2}\right)^{s} u(x) \overline{v(x)} d x .
$$

We write $\mathcal{L}^{2}$ for $\mathcal{L}^{2,0}$ and $\|u\|_{0}=\|u\|_{0,0}$. We also write $\mathcal{H}^{p}$ for $\mathcal{H}^{p, 0}$ and, when needed for clarity, $\|u\|_{\mathcal{H}^{p}}=\|u\|_{p, 0}$. The scalar product is then denoted by $(\cdot, \cdot)$. We do not distinguish in this scalar product between scalar and vector-valued functions; that will be clear from the context.

Let $K>0$ be an integer. For functions valued in $\mathbb{C}^{K}$, if needed for clarity, we denote, as has already been done above for $\mathcal{L}^{2}$,

$$
\mathcal{H}^{p, s}\left(\mathbb{R}^{n}, \mathbb{C}^{K}\right)=\mathcal{H}^{p, s}\left(\mathbb{R}^{n}\right) \bigotimes \mathbb{C}^{K} .
$$

For negative (integer) indices $-p$ we denote by $\left\{\mathcal{H}^{-p, s},\|\cdot\|_{-p, s}\right\}$ the dual space of $\mathcal{H}^{p,-s}$. In particular, observe that any function $f \in \mathcal{H}^{-1, s}$ can be represented (not uniquely) as

$$
f=f_{0}+\sum_{k=1}^{n} i^{-1} \frac{\partial}{\partial x_{k}} f_{k}, \quad f_{k} \in \mathcal{L}^{2, s}, \quad 0 \leq k \leq n .
$$

On the side of the (Fourier) transformed functions we shall need Sobolev spaces of any real order. The Fourier transform (2.1) carries weighted- $L^{2}$ spaces to Sobolev spaces (and vice-versa).

We let $\mathcal{H}^{\theta}=\mathcal{H}^{\theta}\left(\mathbb{R}^{n}\right), \theta \in \mathbb{R}$ be the Sobolev space (based on $L^{2}\left(\mathbb{R}^{n}\right)$ ) of order $\theta$ of functions obtained as Fourier transforms, namely,

$$
\mathcal{H}^{\theta}=\left\{\widehat{u} \quad / \quad u \in \mathcal{L}^{2, \theta}\left(\mathbb{R}^{n}\right), \quad\|\widehat{u}\|_{\mathcal{H}^{\theta}}=\|u\|_{0, \theta}\right\} .
$$

Of course for an integer $\theta=p$ the definitions of $\mathcal{H}^{p}$ and $\mathcal{H}^{\theta}$ are consistent, noting that the latter is used in the Fourier $\xi$-space.

Remark that the more general (weighted) spaces $\mathcal{H}^{\theta, s}$ can be defined, for example, by interpolating between $\mathcal{H}^{p, s}$ with integer values of $p$, but we shall make no use of such (weighted) spaces.

In our study we rely heavily on the trace lemma for functions in $\mathcal{H}^{\theta}\left(\mathbb{R}^{n}\right)$ [5] Proposition 6.3],

Lemma 3.1. Let $\widehat{h} \in \mathcal{H}^{\theta}\left(\mathbb{R}^{n}\right), n \geq 3, \frac{1}{2}<\theta<\frac{3}{2}$. Denote the sphere of radius $r$ by

$$
S_{r}=\left\{\xi \in \mathbb{R}^{n} /|\xi|=r\right\} .
$$

Then

$$
\int_{S_{r}}|\widehat{h}|^{2} d \Sigma_{r} \leq C \min \left(1, r^{2 \theta-1}\right)\|\widehat{h}\|_{\mathcal{H}^{\theta}}^{2}, r>0
$$

where $C>0$ is independent of $\widehat{h}, r$, and $d \Sigma_{r}$ is the Lebesgue surface measure on $S_{r}$. 
Furthermore, the family of trace maps $\left\{\Phi_{r}: \mathcal{H}^{\theta}\left(\mathbb{R}^{n}\right) \rightarrow \mathcal{L}^{2}\left(S_{1}\right), r \in \mathbb{R}_{+}\right\}$given

$$
\Phi_{r} \widehat{h}(\omega)=r^{\frac{n-1}{2}} \widehat{h}(r \omega), \quad \omega \in S_{1}, r \in \mathbb{R}_{+},
$$

is locally Hölder continuous, in the following sense.

For a closed interval $[a, b] \subseteq \mathbb{R}_{+}$there exist constants $C>0$ and $\alpha \in(0,1)$ such that

$$
\int_{S_{1}}\left|\Phi_{r_{2}} \widehat{h}(\omega)-\Phi_{r_{1}} \widehat{h}(\omega)\right|^{2} d \Sigma_{1} \leq C\|\widehat{h}\|_{\mathcal{H}^{\theta}}^{2}\left|r_{2}-r_{1}\right|^{\alpha}, \quad r_{1}, r_{2} \in[a, b] .
$$

Notation. For any two Banach spaces we use $B(X, Y)$ to denote the space of linear bounded operators from $X$ to $Y$, equipped with the uniform operator topology.

\section{SPECTRAL STRUCTURE of the UNPERTURBED DIRAC and MAXWELL OPERATORS}

In this section, we study in detail the spectral properties of the free Dirac and Maxwell operators operators, as introduced in Section 2. This will provide a prelude to the general case that we defer to Section 5 .

4.1. THE FREE DIRAC OPERATOR. We first consider the spectral density of the free Dirac operator, with suitable estimates in weighted Sobolev spaces. These estimates allow us to derive the Limiting Absorption Principle for this operator (Theorem 4.2 below).

Recall the definition (2.10) of the transformation $\mathcal{G}_{m}$.

Observe that in view of Equation (2.11) the operator $\mathcal{G}_{m} H_{m} \mathcal{G}_{m}^{-1}$ is reduced by the two (complementary) orthogonal subspaces $X_{ \pm} \subseteq \mathcal{L}^{2}\left(\mathbb{R}^{3}, \mathbb{C}^{4}\right)$ given by

$$
\begin{aligned}
& X_{+}=\left\{f \in \mathcal{L}^{2}\left(\mathbb{R}^{3}, \mathbb{C}^{4}\right) / \text { the two last components of } \mathcal{G}_{m} f(\xi) \text { vanish }\right\} \\
& X_{-}=\left\{f \in \mathcal{L}^{2}\left(\mathbb{R}^{3}, \mathbb{C}^{4}\right) / \text { the two first components of } \mathcal{G}_{m} f(\xi) \text { vanish }\right\} .
\end{aligned}
$$

In order to determine the domain of the self-adjoint operator $H_{m}$ we repeat the discussion in [30, Section V.5.4].

From (2.9) we obtain the coercivity property (in each reducing subspace)

$$
\begin{array}{r}
\left|<M_{m}(\xi) \widehat{f}(\xi), \widehat{f}(\xi)>_{\mathbb{C}^{4}}\right|=\left|<U_{m}^{*}(\xi) M_{m}(\xi) U_{m}(\xi) U_{m}^{*}(\xi) \widehat{f}(\xi), U_{m}^{*}(\xi) \widehat{f}(\xi)>_{\mathbb{C}^{4}}\right| \\
\geq|\xi||\widehat{f}(\xi)|^{2}, \quad f \in X_{ \pm}, \xi \in \mathbb{R}^{3} .
\end{array}
$$

It follows that the domain of $H_{m}$ in each reducing subspace, hence in the full space $\mathcal{L}^{2}\left(\mathbb{R}^{3}, \mathbb{C}^{4}\right)$, is

$$
\operatorname{Dom}\left(H_{m}\right)=\mathcal{H}^{1}\left(\mathbb{R}^{3}, \mathbb{C}^{4}\right),
$$

and its spectrum (that is absolutely continuous) is

$$
\operatorname{spec}\left(H_{m}\right)=\mathbb{R} \backslash(-m, m)
$$

(it is of course $\mathbb{R}$ if $m=0$ ).

Recall that by Equation (2.11) the operator $H_{m}$ can be diagonalized with

$$
\lambda_{ \pm}(r)= \pm \sqrt{r^{2}+m^{2}}
$$

Let $E_{m}(\lambda)$ be the spectral family associated with $H_{m}$. 
As is customary we use $\chi_{B}$ as the indicator function for a set $B \subseteq \mathbb{R}^{3}$, namely, $\chi_{B}(\xi)=1$ (resp. $\left.\chi_{B}(\xi)=0\right)$ if $\xi \in B($ resp. $\xi \notin B)$.

It is easily seen that if we confine $f \in C_{0}^{\infty}\left(\mathbb{R}^{3}, \mathbb{C}^{4}\right)$ then for $\lambda>m$ we have

$$
\left(E_{m}(\lambda) f, f\right)=\left(\left(\begin{array}{cc}
\chi_{\lambda_{+}(\xi) \leq \lambda} I_{2} & O_{2} \\
O_{2} & I_{2}
\end{array}\right) \mathcal{G}_{m} f, \mathcal{G}_{m} f\right)
$$

where the right-hand side is the scalar product in $\mathcal{L}_{\xi}^{2}\left(\mathbb{R}^{3}, \mathbb{C}^{4}\right)$.

Differentiating the last equality (assuming $f, g$ to be sufficiently regular), we get (with $d \Sigma_{r}$ being the Lebesgue surface measure on the sphere of radius $r>0$ ),

$$
\frac{d}{d \lambda}\left(E_{m}(\lambda) f, f\right)=\frac{\lambda}{\sqrt{\lambda^{2}-m^{2}}} \int_{|\xi|=\sqrt{\lambda^{2}-m^{2}}}\left|\left(\mathcal{G}_{m} f\right)_{+}(\xi)\right|^{2} d \Sigma \sqrt{\lambda^{2}-m^{2}},
$$

where $\left(\mathcal{G}_{m} f\right)_{+}$is a 2 -vector consisting of the first two components of $\mathcal{G}_{m} f$.

An analogous equation is clearly valid in the case $\lambda<-m$.

Since

$$
\left|\left(\mathcal{G}_{m} f\right)_{+}(\xi)\right| \leq\left|\left(\mathcal{G}_{m} f\right)(\xi)\right| \leq|\widehat{f}(\xi)|,
$$

we conclude from Lemma 3.1 and the definition 3.5 of the space $\mathcal{H}^{s}$ that, for any $|\lambda|>m$ and $\frac{1}{2}<s<\frac{3}{2}$, there exists an operator

$$
A_{m}(\lambda) \in B\left(\mathcal{L}^{2, s}\left(\mathbb{R}^{3}, \mathbb{C}^{4}\right), \mathcal{L}^{2,-s}\left(\mathbb{R}^{3}, \mathbb{C}^{4}\right)\right)
$$

such that

$$
\begin{aligned}
< & A_{m}(\lambda) f, f>=\frac{d}{d \lambda}\left(E_{m}(\lambda) f, f\right) \\
& \leq C \min \left(\frac{|\lambda|}{\sqrt{\lambda^{2}-m^{2}}},|\lambda|\left(\lambda^{2}-m^{2}\right)^{s-1}\right)\|\widehat{f}\|_{\mathcal{H}^{s}}^{2} \\
& =C \min \left(\frac{|\lambda|}{\sqrt{\lambda^{2}-m^{2}}},|\lambda|\left(\lambda^{2}-m^{2}\right)^{s-1}\right)\|\widehat{f}\|_{\mathcal{H}^{s}}^{2}
\end{aligned}
$$

where $<,>$ is the $\left(\mathcal{L}^{2,-s}\left(\mathbb{R}^{3}, \mathbb{C}^{4}\right), \mathcal{L}^{2, s}\left(\mathbb{R}^{3}, \mathbb{C}^{4}\right)\right)$ pairing.

Proposition 4.1. (1) Let $s>\frac{1}{2}$. Then the weak derivative $A_{m}(\lambda)=\frac{d}{d \lambda}\left(E_{m}(\lambda)\right)$ is locally bounded and locally Hölder continuous for $|\lambda|>m$, with respect to the uniform operator topology of $B\left(\mathcal{L}^{2, s}\left(\mathbb{R}^{3}, \mathbb{C}^{4}\right), \mathcal{L}^{2,-s}\left(\mathbb{R}^{3}, \mathbb{C}^{4}\right)\right)$.

(2) Let $s>1$. Then the weak derivative $A_{m}(\lambda)=\frac{d}{d \lambda}\left(E_{m}(\lambda)\right)$ is uniformly bounded and uniformly Hölder continuous for $\lambda \in \mathbb{R}$, with respect to the uniform operator topology of $B\left(\mathcal{L}^{2, s}\left(\mathbb{R}^{3}, \mathbb{C}^{4}\right), \mathcal{L}^{2,-s}\left(\mathbb{R}^{3}, \mathbb{C}^{4}\right)\right)$.

Proof. Note that the topology of $B\left(\mathcal{L}^{2, s}\left(\mathbb{R}^{3}, \mathbb{C}^{4}\right), \mathcal{L}^{2,-s}\left(\mathbb{R}^{3}, \mathbb{C}^{4}\right)\right)$ becomes weaker as $s$ grows, so without loss of generality we can assume $s<\frac{3}{2}$, so the estimate (4.7) can be used.

Consider the expression for the spectral derivative, Equation (4.6). Using the expression (2.10), we can rewrite it explicitly as

$$
<A_{m}(\lambda) f, f>=\frac{\lambda}{\sqrt{\lambda^{2}-m^{2}}} \int_{|\xi|=\sqrt{\lambda^{2}-m^{2}}}\left|\left[U_{m}^{*}(\xi) \widehat{f}(\xi)\right]_{+}\right|^{2} d \Sigma_{\sqrt{\lambda^{2}-m^{2}}}
$$


We now estimate the right-hand side of Equation (4.8) in $\mathcal{H}^{\bar{s}}$ with $\bar{s}>2$. By the Sobolev embedding theorem,

$$
|\widehat{f}(\xi)| \leq C\|\widehat{f}\|_{\mathcal{H}^{\bar{s}}}, \quad \xi \in \mathbb{R}^{3} .
$$

Thus

$$
\left.\left|\int_{|\xi|=\sqrt{\lambda^{2}-m^{2}}}\right|\left[U_{m}^{*}(\xi) \widehat{f}(\xi)\right]_{+}\right|^{2} d \Sigma_{\sqrt{\lambda^{2}-m^{2}}} \mid \leq C\left(\lambda^{2}-m^{2}\right)\|f\|_{0, \bar{s}}^{2}, \bar{s}>2,
$$

and from (4.8) we obtain in this case

$$
<A_{m}(\lambda) f, f>\leq C \lambda\left(\lambda^{2}-m^{2}\right)^{\frac{1}{2}}\|f\|_{0, \bar{s}}^{2}, \bar{s}>2 .
$$

Furthermore, using the explicit form (2.12) of $U_{m}(\xi)$ and the Sobolev embeddding theorem we have, in addition to (4.9), also

$$
\left|\nabla_{\xi}\left[U_{m}^{*}(\xi) \widehat{f}(\xi)\right]\right| \leq C\|\widehat{f}\|_{\mathcal{H}^{s}}, \quad \xi \in \mathbb{R}^{3}, \bar{s}>3 .
$$

Rewriting (4.8) in the form

$$
<A_{m}(\lambda) f, f>=\frac{\lambda}{\sqrt{\lambda^{2}-m^{2}}} \int_{|\omega|=1}\left|\left[U_{m}^{*}\left(\sqrt{\lambda^{2}-m^{2}} \omega\right) \widehat{f}\left(\sqrt{\lambda^{2}-m^{2}} \omega\right)\right]_{+}\right|^{2}\left(\lambda^{2}-m^{2}\right) d \Sigma_{1},
$$

we can differentiate to obtain, for $|\lambda|>m$,

$$
\left|\frac{d}{d \lambda}<A_{m}(\lambda) f, f>\right| \leq C\left(\lambda^{2}-m^{2}\right)^{-\frac{1}{2}}\|f\|_{0, \bar{s}}^{2}, \bar{s}>3 .
$$

Since the right-hand side is uniformly locally integrable in $\lambda \in \mathbb{R} \backslash(-m, m)$, we conclude that the operator-valued function

$$
A_{m}(\lambda) \in B\left(\mathcal{L}^{2, \bar{s}}\left(\mathbb{R}^{3}, \mathbb{C}^{4}\right), \mathcal{L}^{2,-\bar{s}}\left(\mathbb{R}^{3}, \mathbb{C}^{4}\right)\right), \quad|\lambda| \geq m, \bar{s}>3,
$$

is uniformly Hölder continuous (and vanishes at $|\lambda|=m$ ).

Interpolating this estimate with the boundedness estimates (4.7) we conclude that

- For $s>\frac{1}{2}$ the operator-valued function

$$
A_{m}(\lambda) \in B\left(\mathcal{L}^{2, s}\left(\mathbb{R}^{3}, \mathbb{C}^{4}\right), \mathcal{L}^{2,-s}\left(\mathbb{R}^{3}, \mathbb{C}^{4}\right)\right), \quad|\lambda|>m,
$$

is locally bounded and locally Hölder continuous .

- For $s>1$ the operator-valued function

$$
A_{m}(\lambda) \in B\left(\mathcal{L}^{2, s}\left(\mathbb{R}^{3}, \mathbb{C}^{4}\right), \mathcal{L}^{2,-s}\left(\mathbb{R}^{3}, \mathbb{C}^{4}\right)\right), \quad|\lambda| \geq m,
$$

is uniformly bounded and uniformly Hölder continuous in $\lambda \in \mathbb{R}$ (and vanishes for $\lambda \in[-m, m])$.

This concludes the proof of the proposition.

We can state a slightly more general fact by taking the norms of $f$ and $g$ below in different weighted spaces. In fact, suppose that $f, g$ are smooth and compactly supported. Since the bilinear form $\left\langle A_{m}(\lambda) \cdot, \cdot\right\rangle$ is nonegative, applying the Cauchy-Schwarz inequality yields, for any $|\lambda|>m$ and $s, l>\frac{1}{2}$, 
$(4.13)$

$$
\begin{aligned}
\left|\frac{d}{d \lambda}\left(E_{m}(\lambda) f, g\right)\right|=\mid< & A_{m}(\lambda) f, g>\mid \leq \quad<A_{m}(\lambda) f, f>^{\frac{1}{2}} \cdot<A_{m}(\lambda) g, g>^{\frac{1}{2}} \\
& \leq C \min \left(\frac{|\lambda|}{\sqrt{\lambda^{2}-m^{2}}},|\lambda|\left(\lambda^{2}-m^{2}\right)^{\frac{s+l}{2}-1}\right)\|\widehat{f}\|_{\mathcal{H}^{s}}\|\widehat{g}\|_{\mathcal{H}^{l}} \\
& =C \min \left(\frac{|\lambda|}{\sqrt{\lambda^{2}-m^{2}}},|\lambda|\left(\lambda^{2}-m^{2}\right)^{\frac{s+l}{2}-1}\right)\|f\|_{0, s}\|g\|_{0, l} .
\end{aligned}
$$

It is obvious that in the inequality above, the first $<,>$ is the $\left(\mathcal{L}^{2,-s}\left(\mathbb{R}^{3}, \mathbb{C}^{4}\right), \mathcal{L}^{2, s}\left(\mathbb{R}^{3}, \mathbb{C}^{4}\right)\right)$ pairing, while the second is the $\left(\mathcal{L}^{2,-l}\left(\mathbb{R}^{3}, \mathbb{C}^{4}\right), \mathcal{L}^{2, l}\left(\mathbb{R}^{3}, \mathbb{C}^{4}\right)\right)$ pairing.

The general theory [5, Section 3] now yields the Limiting Absorption Principle (LAP) for the unperturbed Dirac operator.

Theorem 4.2. Let $R_{m}(z)=\left(H_{m}-z\right)^{-1}$, Im $z \neq 0$. For any $s, l>\frac{1}{2}$ the limits

$$
R_{m}^{ \pm}(\mu)=\lim _{\varepsilon \downarrow 0} R_{m}(\mu \pm i \varepsilon), \mu \in \mathbb{R} \backslash[-m, m],
$$

exist in the uniform operator topology of $B\left(\mathcal{L}^{2, s}\left(\mathbb{R}^{3}, \mathbb{C}^{4}\right), \mathcal{H}^{1,-l}\left(\mathbb{R}^{3}, \mathbb{C}^{4}\right)\right)$.

If $s, l>1$ then the limits in (4.14) exist for all $\mu \in \mathbb{R}$, or, otherwise stated, they are continuous across the thresholds at $\mu= \pm m$.

Furthermore, in both cases the limit functions $R_{m}^{ \pm}(\mu)$ are locally bounded and locally Hölder continuous (in their respective domains) with respect to the uniform operator topology.

Proof. As already pointed out, the properties of $A_{m}(\lambda)$, as given in Proposition 4.1 enable us to invoke the general theory and obtain the result in the operator setting of $B\left(\mathcal{L}^{2, s}\left(\mathbb{R}^{3}, \mathbb{C}^{4}\right), \mathcal{L}^{2,-l}\left(\mathbb{R}^{3}, \mathbb{C}^{4}\right)\right)$.

In order to complete the proof we need to show that it is possible to replace $\mathcal{L}^{2,-l}\left(\mathbb{R}^{3}, \mathbb{C}^{4}\right)$ by $\mathcal{H}^{1,-l}\left(\mathbb{R}^{3}, \mathbb{C}^{4}\right)$.

Take $f \in \mathcal{L}^{2, s}\left(\mathbb{R}^{3}, \mathbb{C}^{4}\right)$, so that by the already established result, the limit

$$
R_{m}^{ \pm}(\mu) f=\lim _{\varepsilon \downarrow 0} R_{m}(\mu \pm i \varepsilon) f, \mu \in \mathbb{R} \backslash[-m, m],
$$

exists in $\mathcal{L}^{2,-l}\left(\mathbb{R}^{3}, \mathbb{C}^{4}\right)$.

We have

$$
\left(H_{m}-\mu\right) R_{m}(\mu \pm i \varepsilon) f=f \pm i \varepsilon R_{m}(\mu \pm i \varepsilon) f
$$

and since $\left\{R_{m}(\mu \pm i \varepsilon) f, 0<\varepsilon<1\right\}$ is uniformly bounded in $\mathcal{L}^{2,-l}\left(\mathbb{R}^{3}, \mathbb{C}^{4}\right)$, we obtain the limit (in this space)

$$
\lim _{\varepsilon \downarrow 0} H_{m} R_{m}(\mu \pm i \varepsilon) f=f+\mu R_{m}^{ \pm}(\mu) f .
$$

Note that $H_{m}$ is densely defined and closable in $\mathcal{L}^{2,-l}\left(\mathbb{R}^{3}, \mathbb{C}^{4}\right)$ and in fact, in view of the coercivity (4.2) its graph norm in this space is equivalent to the norm of $\mathcal{H}^{1,-l}\left(\mathbb{R}^{3}, \mathbb{C}^{4}\right)$.

Retaining the same notation for its closure, we get

$$
H_{m} R_{m}^{ \pm}(\mu) f=f+\mu R_{m}^{ \pm}(\mu) f,
$$

so that indeed

$$
R_{m}^{ \pm}(\mu) f \in \mathcal{H}^{1,-l}\left(\mathbb{R}^{3}, \mathbb{C}^{4}\right) .
$$


Remark 4.3. - The first part of Theorem 4.2 (namely, $s, l>\frac{1}{2}$ and avoiding the thresholds) was obtained in [3, 51. Both papers made use of the LAP for the Schrödinger operator, by way of formula (2.8).

- Note that for the second part of the theorem it suffices to assume $s, l>\frac{1}{2}$ with $s+l>2$.

- In Proposition 4.5 below we give a somewhat different argument for the proof of (4.15), based on the fact that $H_{m}$ is a constant coefficient operator.

We shall now extend this theorem to more general function spaces. We take $s, l>1$.

Let $g \in \mathcal{H}^{1, l}\left(\mathbb{R}^{3}, \mathbb{C}^{4}\right), f \in \mathcal{H}^{-1, s}\left(\mathbb{R}^{3}, \mathbb{C}^{4}\right)$, where $f$ has a representation of the form (3.4), with $f_{k} \in \mathcal{L}^{2, s}\left(\mathbb{R}^{3}, \mathbb{C}^{4}\right), 0 \leq k \leq 3$.

Equation (4.6) can be extended (at least formally) to yield

$$
\begin{gathered}
<A_{m}(\lambda)\left[f_{0}+i^{-1} \sum_{k=1}^{3} \frac{\partial}{\partial x_{k}} f_{k}\right], g> \\
=\frac{\lambda}{\sqrt{\lambda^{2}-m^{2}}} \int_{|\xi|^{2}=\lambda^{2}-m^{2}}<\left(\mathcal{G}_{m} \widehat{f_{0}}\right)_{+}(\xi)+\sum_{k=1}^{3} \xi_{k}\left(\mathcal{G}_{m} \widehat{f_{k}}\right)_{+}(\xi),\left(\mathcal{G}_{m} \widehat{g}\right)_{+}(\xi)>_{\mathbb{C}^{2}} d \Sigma_{\sqrt{\lambda^{2}-m^{2}}} \\
f \in \mathcal{H}^{-1, s}, g \in \mathcal{H}^{1, l} .
\end{gathered}
$$

Observe that this definition makes good sense even though the representation (3.4) is not unique, since

$$
f=f_{0}+\sum_{k=1}^{3} i^{-1} \frac{\partial}{\partial x_{k}} f_{k}=\tilde{f}_{0}+\sum_{k=1}^{3} i^{-1} \frac{\partial}{\partial x_{k}} \tilde{f}_{k},
$$

implies

$$
\widehat{f_{0}}(\xi)+\sum_{k=1}^{3} \xi_{k} \widehat{f}_{k}(\xi)=\widehat{\tilde{f}}_{0}(\xi)+\sum_{k=1}^{3} \xi_{k} \widehat{\widetilde{f}}_{k}(\xi)
$$

(as tempered distributions).

Proposition 4.4. Equation (4.16) can indeed be used to define an operator (for which we retain the same notation)

$$
A_{m}(\lambda) \in B\left(\mathcal{H}^{-1, s}\left(\mathbb{R}^{3}, \mathbb{C}^{4}\right), \mathcal{H}^{-1,-l}\left(\mathbb{R}^{3}, \mathbb{C}^{4}\right)\right) .
$$

In this setting $<,>$ is the $\left(\mathcal{H}^{-1,-l}, \mathcal{H}^{1, l}\right)$ pairing and $|\lambda|>m$.

Proof. To estimate the operator-norm of $A_{m}(\lambda)$ as given in (4.16) we use, for $1 \leq$ $k \leq 3$, the estimate (4.13), in the form

$$
\begin{aligned}
\left|<A_{m}(\lambda) \frac{\partial}{\partial x_{k}} f_{k}, g>\right|=\frac{|\lambda|}{\sqrt{\lambda^{2}-m^{2}}}\left|\int_{|\xi|^{2}=\lambda^{2}-m^{2}}<\left(\mathcal{G}_{m} \widehat{f_{k}}\right)_{+}(\xi), \xi_{k}\left(\mathcal{G}_{m} \widehat{g}\right)_{+}(\xi)>_{\mathbb{C}^{2}} d \Sigma_{\sqrt{\lambda^{2}-m^{2}}}\right| \\
\leq C \min \left(\frac{|\lambda|}{\sqrt{\lambda^{2}-m^{2}}},|\lambda|\left(\lambda^{2}-m^{2}\right)^{\frac{s+l}{2}-1}\right)\left\|\widehat{f_{k}}\right\|_{\mathcal{H}^{s}}\left\|\widehat{\xi_{k} g}\right\|_{\mathcal{H}^{l}},
\end{aligned}
$$


so from (4.16) we obtain

(4.17)

$$
\begin{gathered}
\left|<A_{m}(\lambda) f, g>\right| \\
\leq C \min \left(\frac{|\lambda|}{\sqrt{\lambda^{2}-m^{2}}},|\lambda|\left(\lambda^{2}-m^{2}\right)^{\frac{s+l}{2}-1}\right)\|f\|_{-1, s}\|g\|_{1, l}, \quad f \in \mathcal{H}^{-1, s}, g \in \mathcal{H}^{1, l}, s, l>1 .
\end{gathered}
$$

Theorem 4.2 can now be enhanced to yield

Proposition 4.5. The operator-valued function $R_{m}(z)$ is well-defined (and analytic) for nonreal $z$ in the following functional setting.

$$
z \rightarrow R_{m}(z) \in B\left(\mathcal{H}^{-1, s}\left(\mathbb{R}^{3}, \mathbb{C}^{4}\right), \mathcal{L}^{2,-l}\left(\mathbb{R}^{3}, \mathbb{C}^{4}\right)\right) .
$$

where $s, l>1$.

Furthermore, it can be extended continuously from $\mathbb{C}^{ \pm}$to $\overline{\mathbb{C}^{ \pm}}$, in this uniform operator topology. The limiting values (denoted again by $R_{m}^{ \pm}(\lambda)$ ) are Hölder continuous in the same topology.

The extended function satisfies

$$
\left(H_{m}-z\right) R_{m}(z) f=f, \quad f \in \mathcal{H}^{-1, s}\left(\mathbb{R}^{3}, \mathbb{C}^{4}\right), \quad z \in \overline{\mathbb{C}^{ \pm}},
$$

where for $z=\lambda \in \mathbb{R}, \quad R_{m}(z)=R_{m}^{ \pm}(\lambda)$.

Proof. By the estimate (4.17), we get readily $R_{m}(z) \in B\left(\mathcal{H}^{-1, s}\left(\mathbb{R}^{3}, \mathbb{C}^{4}\right), \mathcal{H}^{-1,-l}\left(\mathbb{R}^{3}, \mathbb{C}^{4}\right)\right)$ if $\operatorname{Im} z \neq 0$, as well as the analyticity of the map $z \hookrightarrow R_{m}(z), \operatorname{Im} z \neq 0$. Furthermore, the extension to $\operatorname{Im} z=0$ is carried out as in [4, Section 4].

Equation (4.19) is obvious if $\operatorname{Im} z \neq 0$ and $f \in \mathcal{L}^{2, s}$. By the density of $\mathcal{L}^{2, s}$ in $\mathcal{H}^{-1, s}$, the continuity of $R_{m}(z)$ on $\mathcal{H}^{-1, s}$ and the continuity of $H_{m}-z$ (in the sense of distributions), we can extend it to all $f \in \mathcal{H}^{-1, s}$.

As $z \rightarrow \lambda \pm i \cdot 0$ we have $R_{m}(z) f \rightarrow R_{m}^{ \pm}(\lambda) f$ in $\mathcal{H}^{-1,-l}$. Applying the (constant coefficient) operator $H_{m}-z$ yields, in the sense of distributions, $f=\left(H_{m}-\right.$ z) $R_{m}(z) f \rightarrow\left(H_{m}-\lambda\right) R_{m}^{ \pm}(\lambda) f$ which establishes (4.19) also for $\operatorname{Im} z=0$.

Finally, the established continuity of $z \hookrightarrow R_{m}(z) \in B\left(\mathcal{H}^{-1, s}, \mathcal{H}^{-1,-l}\right)$ up to the real boundary and Equation (4.19) imply the continuity of the map $z \hookrightarrow$ $H_{m} R_{m}(z) \in B\left(\mathcal{H}^{-1, s}\left(\mathbb{R}^{3}, \mathbb{C}^{4}\right), \mathcal{H}^{-1,-l}\left(\mathbb{R}^{3}, \mathbb{C}^{4}\right)\right)$.

The stronger continuity claim (4.18) follows since the norm of $\mathcal{L}^{2,-l}$ is equivalent to the graph-norm of $H_{m}$ as a map of $\mathcal{H}^{-1,-l}$ to itself.

Remark 4.6. Note that we could actually take $s, l>\frac{1}{2}$ with $s+l>2$. This is identical to Proposition 2.4 in [12], except that here we obtain the Hölder continuity of the limiting values.

4.2. THE FREE MAXWELL OPERATOR. In Subsection 2.2 we introduced the Maxwell operator $L_{\text {maxwell }}$. It is a constant coefficient differential operator and its symbol $M_{\text {maxwell }}(\xi)$ is Hermitian. Thus, it can be realized as a self-adjoint operator in $\mathcal{L}^{2}\left(\mathbb{R}^{3}, \mathbb{C}^{6}\right)$, for which we retain the same notation.

The spectrum of the operator is readily seen to be

$$
\operatorname{spec}\left(L_{\text {maxwell }}\right)=\mathbb{R} \text {. }
$$

The spectrum is absolutely continuous except for the point $\lambda=0$. In view of Corollary 2.4 the eigenspace associated with the zero eigenvalue is given by

$$
\operatorname{ker}\left(L_{\text {maxwell }}\right)=\left\{f \in \mathcal{L}^{2}\left(\mathbb{R}^{3}, \mathbb{C}^{6}\right) / \widehat{f}(\xi) \in \operatorname{span}\left\{\Upsilon_{0}\right\}, 0 \neq \xi \in \mathbb{R}^{3}\right\} .
$$


We now consider the spectral density and the Limiting Absorption Principle for the Maxwell operator, in the setting of weighted Sobolev spaces. The treatment is quite analogous to that of the Dirac operator and we discuss it briefly, focusing on the aspects of difference between the two cases. As in the Dirac operator case, we obtain detailed estimates at the threshold energy $\lambda=0$. Such estimates are needed for global spacetime estimates (Subsection 7.2). Note that the Maxwell operator is not elliptic (nor bounded from below), so suitable care is needed with respect to its domain of definition .

In addition to the kernel expressed in (4.21) we define two other (complementary) subspaces, as follows.

$$
X_{ \pm}=\left\{f \in \mathcal{L}^{2}\left(\mathbb{R}^{3}, \mathbb{C}^{6}\right) / \widehat{f}(\xi) \in \operatorname{span}\left\{\Upsilon_{ \pm}\right\}, 0 \neq \xi \in \mathbb{R}^{3}\right\} .
$$

Note (compare (4.1)) that these subspaces can also be expressed as

$$
\begin{aligned}
& X_{+}=\left\{f \in \mathcal{L}^{2}\left(\mathbb{R}^{3}, \mathbb{C}^{6}\right) / \text { the four last components of } \mathcal{T}_{0} f(\xi) \text { vanish }\right\}, \\
& X_{-}=\left\{f \in \mathcal{L}^{2}\left(\mathbb{R}^{3}, \mathbb{C}^{6}\right) / \text { the four first components of } \mathcal{T}_{0} f(\xi) \text { vanish }\right\},
\end{aligned}
$$

where the transformation $\mathcal{T}_{0}$ is defined in (2.18).

Observe that these subspaces are those containing the "TE, TM" modes (Remark 2.6).

These subspaces are clearly reducing for $L_{\text {maxwell }}$. From (2.17) we obtain the (partial) coercivity property

$$
\begin{aligned}
\mid<M_{\text {maxwell }}(\xi) \widehat{f}(\xi), \widehat{f}(\xi) & >_{\mathbb{C}^{6}}|=|<M_{\text {maxwell }}(\xi) \mathcal{T}_{0} f(\xi), \mathcal{T}_{0} f(\xi)>_{\mathbb{C}^{6}} \mid \\
& \geq|\xi|\left|\mathcal{T}_{0} f(\xi)\right|^{2}=\left.|\xi \|| \widehat{f}(\xi)\right|^{2}, \quad \xi \in \mathbb{R}^{3}, f \in X_{ \pm} .
\end{aligned}
$$

These facts enable us to give an explicit expression for the domain of $L_{\text {maxwell }}$.

Claim 4.7. The domain of $L_{\text {maxwell }}$, as a self-adjoint operator in $\mathcal{L}^{2}\left(\mathbb{R}^{3}, \mathbb{C}^{6}\right)$, is given by

$$
\operatorname{Dom}\left(L_{\text {maxwell }}\right)=\operatorname{ker}\left(L_{\text {maxwell }}\right) \oplus\left(X_{+} \cap \mathcal{H}^{1}\left(\mathbb{R}^{3}, \mathbb{C}^{6}\right)\right) \oplus\left(X_{-} \cap \mathcal{H}^{1}\left(\mathbb{R}^{3}, \mathbb{C}^{6}\right)\right) .
$$

The eigenvalues of the symbol $M_{\text {maxwell }}(\xi)$ are

$$
\lambda_{ \pm}(\xi)= \pm|\xi| \text {. }
$$

Let $F(\lambda)$ be the spectral family associated with $L_{\text {maxwell }}$.

It is easily seen that if we confine $f \in C_{0}^{\infty}\left(\mathbb{R}^{3}, \mathbb{C}^{6}\right)$ then for $\lambda>0$ we have

$$
(F(\lambda) f, f)=\left(\left(\begin{array}{ccc}
\chi_{\lambda_{+}(\xi) \leq \lambda} I_{2} & O_{2} & O_{2} \\
O_{2} & O_{2} & O_{2} \\
O_{2} & O_{2} & I_{2}
\end{array}\right) \mathcal{T}_{0} f, \mathcal{T}_{0} f\right),
$$

where the right-hand side is the scalar product in $\mathcal{L}_{\xi}^{2}\left(\mathbb{R}^{3}, \mathbb{C}^{6}\right)$.

Differentiating the last equality (assuming $f, g$ to be sufficiently regular), we get

$$
\frac{d}{d \lambda}(F(\lambda) f, f)=\int_{|\xi|=\lambda}\left|\left(\mathcal{T}_{0} f\right)_{+}(\xi)\right|^{2} d \Sigma_{\lambda},
$$

where $\left(\mathcal{T}_{0} f\right)_{+}$is a 2 -vector consisting of the first two components of $\mathcal{T}_{0} f$.

An analogous equation is clearly valid in the case $\lambda<0$. 
Since

$$
\left|\left(\mathcal{T}_{0} f\right)_{+}(\xi)\right| \leq\left|\left(\mathcal{T}_{0} f\right)(\xi)\right| \leq|\widehat{f}(\xi)|
$$

we conclude from Lemma 3.1 that, for any $\lambda \in \mathbb{R} \backslash\{0\}$ and $s>\frac{1}{2}$,

$$
\begin{aligned}
\frac{d}{d \lambda}(F(\lambda) f, f) & \leq C \min \left(1,|\lambda|^{2 s-1}\right)\|\widehat{f}\|_{\mathcal{H}^{s}}^{2} \\
& =C \min \left(1,|\lambda|^{2 s-1}\right)\|f\|_{0, s}^{2} .
\end{aligned}
$$

It follows that there exists a map

$$
\widetilde{A}(\lambda) \in B\left(\mathcal{L}^{2, s}\left(\mathbb{R}^{3}, \mathbb{C}^{6}\right), \mathcal{L}^{2,-s}\left(\mathbb{R}^{3}, \mathbb{C}^{6}\right)\right),
$$

so that

$$
\frac{d}{d \lambda}(F(\lambda) f, f)=<\widetilde{A}(\lambda) f, f>,
$$

where $<,>$ is the $\left(\mathcal{L}^{2,-s}\left(\mathbb{R}^{3}, \mathbb{C}^{6}\right), \mathcal{L}^{2, s}\left(\mathbb{R}^{3}, \mathbb{C}^{6}\right)\right)$ pairing.

Proposition 4.8. Let $s>\frac{1}{2}$. Then the weak derivative $\widetilde{A}(\lambda)=\frac{d}{d \lambda}(F(\lambda))$ is locally bounded and locally Hölder continuous for $\lambda \in \mathbb{R} \backslash\{0\}$, with respect to the uniform operator topology of $B\left(\mathcal{L}^{2, s}\left(\mathbb{R}^{3}, \mathbb{C}^{6}\right), \mathcal{L}^{2,-s}\left(\mathbb{R}^{3}, \mathbb{C}^{6}\right)\right)$.

In fact, defining $\widetilde{A}(0)=0$, The function $\widetilde{A}(\lambda)$ is uniformly bounded and uniformly Hölder continuous for $\lambda \in[-r, r]$ in the operator topology, for every $r>0$..

Proof. The proof is quite parallel to that of Proposition 4.1. so we give a rather brief exposition here. We take $\lambda>0$.

Consider the expression for the spectral derivative, Equation (4.27). Using (2.18), we can rewrite it explicitly as

$$
<\widetilde{A}(\lambda) f, f>=\int_{|\xi|=\lambda}\left|\left[V_{0}^{*}(\xi) \widehat{f}(\xi)\right]_{+}\right|^{2} d \Sigma_{\lambda}
$$

The local boundedness of $\widetilde{A}(\lambda) \in B\left(\mathcal{L}^{2, s}\left(\mathbb{R}^{3}, \mathbb{C}^{6}\right), \mathcal{L}^{2,-s}\left(\mathbb{R}^{3}, \mathbb{C}^{6}\right)\right), \lambda \neq 0$, is immediate from (4.28).

In what follows $C>0$ is a generic constant that does not depend on $\xi, \lambda, f$.

Next consider the right-hand side of Equation (4.29) and estimate it in $\mathcal{H}^{\bar{s}}, \bar{s}>2$. In view of the Sobolev embedding theorem (see (3.5) for notation)

$$
|\widehat{f}(\xi)| \leq C\|\widehat{f}\|_{\mathcal{H}^{\bar{s}}}, \quad \xi \in \mathbb{R}^{3} \text {. }
$$

Thus

$$
\left.\left|\int_{|\xi|=\lambda}\right|\left[V_{0}^{*}(\xi) \widehat{f}(\xi)\right]_{+}\right|^{2} d \Sigma_{|\lambda|} \mid \leq C \lambda^{2}\|f\|_{0, \bar{s}}^{2}, \bar{s}>2,
$$

and from 4.29) we obtain in this case

$$
<\widetilde{A}(\lambda) f, f>\leq C \lambda^{2}\|f\|_{0, \bar{s}}^{2}, \bar{s}>2 .
$$

Using the homogeneity of $V_{0}(\xi)$ (of order zero) we have

$$
\mid \nabla_{\xi}\left[V_{0}^{*}(\xi) \mid \leq \frac{C}{|\xi|}, \quad \xi \in \mathbb{R}^{3} \backslash\{0\} .\right.
$$

In conjunction with the Sobolev embeddding theorem we have, in addition to (4.30),

$$
\left|\nabla_{\xi}\left[V_{0}^{*}(\xi) \widehat{f}(\xi)\right]\right| \leq C \max \left(1,|\xi|^{-1}\right)\|\widehat{f}\|_{\mathcal{H}^{\bar{s}}}, \quad \xi \in \mathbb{R}^{3} \backslash\{0\}, \bar{s}>3 .
$$


Rewriting (4.29) in the form

$$
<\widetilde{A}(\lambda) f, f>=\lambda^{2} \int_{|\omega|=1}\left|\left[V_{0}^{*}(\lambda \omega) \widehat{f}(\lambda \omega)\right]_{+}\right|^{2} d \Sigma_{1},
$$

we can differentiate to obtain, for $|\lambda|>0$,

$$
\left|\frac{d}{d \lambda}<\widetilde{A}(\lambda) f, f>\right| \leq C \max \left(\lambda, \lambda^{2}\right)\|f\|_{0, \bar{s}}^{2}, \bar{s}>3 .
$$

The right-hand side is locally integrable in $\lambda \in \mathbb{R}$, hence the operator-valued function

$$
\widetilde{A}(\lambda) \in B\left(\mathcal{L}^{2, \bar{s}}\left(\mathbb{R}^{3}, \mathbb{C}^{6}\right), \mathcal{L}^{2,-\bar{s}}\left(\mathbb{R}^{3}, \mathbb{C}^{6}\right)\right), \quad|\lambda|>0, \bar{s}>3,
$$

is locally Hölder continuous (and vanishes at $|\lambda|=0$ ).

Interpolating between the local boundedness (4.28) for $s>\frac{1}{2}$ and the local Hölder continuity above (for $\bar{s}>3$ ) we obtain the local Hölder continuity for any $s>\frac{1}{2}$.

Remark 4.9. Since $\lambda=0$ is an eigenvalue of $L_{\text {maxwell }}$ there is no spectral derivative there. However, as seen from the estimate (4.28), the weak derivative vanishes as $\lambda \rightarrow 0$.

We can state a slightly more general estimate than (4.28) by taking the norms of $f$ and $g$ below in different weighted spaces. In fact, suppose that $f, g$ are smooth and compactly supported. Then, as in the derivation of the estimate (4.13), for $\lambda \neq 0$, and $s, l>\frac{1}{2}$,

$$
\begin{aligned}
\left|\frac{d}{d \lambda}(F(\lambda) f, g)\right| \leq & <\widetilde{A}(\lambda) f, f>^{\frac{1}{2}} \cdot<\widetilde{A}(\lambda) g, g>^{\frac{1}{2}} \\
\leq & C \min \left(1,|\lambda|^{s+l-1}\right)\|\widehat{f}\|_{\mathcal{H}^{s}}\|\widehat{g}\|_{\mathcal{H}^{l}} \\
= & C \min \left(1,|\lambda|^{s+l-1}\right)\|f\|_{0, s}\|g\|_{0, l} .
\end{aligned}
$$

It is obvious that in the inequality above, the first $<,>$ is the $\left(\mathcal{L}^{2,-s}\left(\mathbb{R}^{3}, \mathbb{C}^{6}\right), \mathcal{L}^{2, s}\left(\mathbb{R}^{3}, \mathbb{C}^{6}\right)\right)$ pairing, while the second is the $\left(\mathcal{L}^{2,-l}\left(\mathbb{R}^{3}, \mathbb{C}^{6}\right), \mathcal{L}^{2, l}\left(\mathbb{R}^{3}, \mathbb{C}^{6}\right)\right)$ pairing.

The general theory [5, Section 3] now yields the Limiting Absorption Principle (LAP) for the unperturbed Maxwell operator.

Theorem 4.10. Let $R_{\text {maxwell }}(z)=\left(L_{\text {maxwell }}-z\right)^{-1}$, Imz $\neq 0$. For any $s, l>\frac{1}{2}$ the limits

$$
R_{\text {maxwell }}^{ \pm}(\mu)=\lim _{\varepsilon \downarrow 0} R_{\text {maxwell }}(\mu \pm i \varepsilon), \mu \in \mathbb{R} \backslash\{0\},
$$

exist in the uniform operator topology of $B\left(\mathcal{L}^{2, s}\left(\mathbb{R}^{3}, \mathbb{C}^{6}\right), \mathcal{L}^{2,-l}\left(\mathbb{R}^{3}, \mathbb{C}^{6}\right)\right)$.

The limits in (4.35) can be extended to $\mu=0$, or, otherwise stated, they are continuous across the eigenvalue at $\mu=0$.

Furthermore, the limit functions $R_{\text {maxwell }}^{ \pm}(\mu), \mu \in \mathbb{R}$, are locally bounded and locally Hölder continuous with respect to the uniform operator topology.

Remark 4.11. Continuing Remark 4.9, notice that the limiting values $R_{\text {maxwell }}^{ \pm}(0)$ are not, of course, limiting values of the resolvent $\left(L_{\text {maxwell }} \pm i \varepsilon\right)^{-1}$ as $\varepsilon \rightarrow 0$. However, on the subspace (of "TE,TM" modes) orthogonal to the kernel we have the following corollary. 
Corollary 4.12. Let $s, l>\frac{1}{2}$. Consider the operator $\mathcal{P}^{\perp} R_{\text {maxwell }}(z)=\mathcal{P}^{\perp}\left(L_{\text {maxwell }}-\right.$ $z)^{-1}, z=\mu \pm i \varepsilon$, where $\mathcal{P}$ is the orthogonal projection on $\operatorname{ker}\left(L_{\text {maxwell }}\right)$ in $\mathcal{L}^{2}\left(\mathbb{R}^{3}, \mathbb{C}^{6}\right)$. Then the limits

$$
\mathcal{P}^{\perp} R_{\text {maxwell }}^{ \pm}(\mu)=\lim _{\varepsilon \downarrow 0} \mathcal{P}^{\perp} R_{\text {maxwell }}(\mu \pm i \varepsilon), \mu \in \mathbb{R},
$$

exist in the uniform operator topology of $B\left(\mathcal{L}^{2, s}\left(\mathbb{R}^{3}, \mathbb{C}^{6}\right), \mathcal{L}^{2,-l}\left(\mathbb{R}^{3}, \mathbb{C}^{6}\right)\right)$.

Furthermore, these limits are Hölder continuous in the same operator topology.

Proof. Indeed, the corollary follows directly from the last part of Proposition 4.8 since the weak derivative $\widetilde{A}(\lambda)=\frac{d}{d \lambda}(F(\lambda)$ ) (extended by $\widetilde{A}(0)=0$ ) is uniformly bounded and uniformly Hölder continuous for $\lambda \in[-r, r]$ in the operator topology, for every $r>0$..

As in the case of the Dirac operator, we can extend the theorem to more general function spaces. We continue to assume $s, l>\frac{1}{2}$.

Let $g \in \mathcal{H}^{1, l}\left(\mathbb{R}^{3}, \mathbb{C}^{6}\right), f \in \mathcal{H}^{-1, s}\left(\mathbb{R}^{3}, \mathbb{C}^{6}\right)$, where $f$ has a representation of the form (3.4), with $f_{k} \in \mathcal{L}^{2, s}\left(\mathbb{R}^{3}, \mathbb{C}^{6}\right), 0 \leq k \leq 3$.

Equation (4.27) can be extended to yield an operator (for which we retain the same notation)

$$
\widetilde{A}(\lambda) \in B\left(\mathcal{H}^{-1, s}\left(\mathbb{R}^{3}, \mathbb{C}^{6}\right), \mathcal{H}^{-1,-l}\left(\mathbb{R}^{3}, \mathbb{C}^{6}\right)\right),
$$

defined by (where now $<,>$ is used for the $\left(\mathcal{H}^{-1,-l}, \mathcal{H}^{1, l}\right)$ pairing and we assume $\lambda>0)$,

$$
\begin{gathered}
<\widetilde{A}(\lambda)\left[f_{0}+i^{-1} \sum_{k=1}^{3} \frac{\partial}{\partial x_{k}} f_{k}\right], g> \\
=\int_{|\xi|=\lambda}<\left(\mathcal{T}_{0} f_{0}\right)_{1}(\xi)+\sum_{k=1}^{3} \xi_{k}\left(\mathcal{T}_{0} f_{k}\right)_{1}(\xi),\left(\mathcal{T}_{0} g\right)_{1}(\xi)>_{\mathbb{C}^{2}} d \Sigma_{\lambda}, \quad f \in \mathcal{H}^{-1, s}, g \in \mathcal{H}^{1, l},
\end{gathered}
$$

that can be rewritten as

$$
\begin{aligned}
< & \widetilde{A}(\lambda)\left[f_{0}+i^{-1} \sum_{k=1}^{3} \frac{\partial}{\partial x_{k}} f_{k}\right], g>=\int_{|\xi|=\lambda}<\left(\mathcal{T}_{0} f_{0}\right)_{1}(\xi),\left(\mathcal{T}_{0} g\right)_{1}(\xi)>_{\mathbb{C}^{2}} d \Sigma_{\lambda} \\
& +\sum_{k=1}^{3} \int_{|\xi|=\lambda}<\left(\mathcal{T}_{0} f_{k}\right)_{1}(\xi) \xi_{k},\left(\mathcal{T}_{0} g\right)_{1}(\xi)>_{\mathbb{C}^{2}} d \Sigma_{\lambda}, \quad f \in \mathcal{H}^{-1, s}, g \in \mathcal{H}^{1, l} .
\end{aligned}
$$

To estimate the operator-norm of $\widetilde{A}(\lambda)$ in this setting we use (4.38) and the considerations leading to (4.34), for $1 \leq k \leq 3$

$$
\begin{aligned}
\mid & <\widetilde{A}(\lambda) \frac{\partial}{\partial x_{k}} f_{k}, g>\mid \\
& \leq C \min \left(1,|\lambda|^{s+l-1}\right)\|f\|_{-1, s}\|g\|_{1, l} \quad, \quad f \in \mathcal{H}^{-1, s}, \quad g \in \mathcal{H}^{1, l},
\end{aligned}
$$

so that, instead of (4.34), we have

$$
|<\widetilde{A}(\lambda) f, g>| \leq C \min \left(1,|\lambda|^{s+l-1}\right)\|f\|_{-1, s}\|g\|_{1, l} .
$$


where $s, l>\frac{1}{2}$.

The following proposition is proved in the same way as Proposition 4.8

Proposition 4.13. Let $s, l>\frac{1}{2}$. Then the weak derivative $\widetilde{A}(\lambda)=\frac{d}{d \lambda}(F(\lambda))$ is locally bounded and locally Hölder continuous for $\lambda \in \mathbb{R} \backslash\{0\}$, with respect to the uniform operator topology of $B\left(\mathcal{H}^{-1, s}\left(\mathbb{R}^{3}, \mathbb{C}^{6}\right), \mathcal{H}^{-1,-l}\left(\mathbb{R}^{3}, \mathbb{C}^{6}\right)\right)$.

When trying to establish the regularization property of the resolvent, in analogy to the Dirac case (Proposition 4.5) we need to take into account the fact that the kernel is nontrivial, so that regularization can only take part in the subspace $\mathcal{P}^{\perp} \mathcal{L}^{2}\left(\mathbb{R}^{3}, \mathbb{C}^{6}\right)$, where $\mathcal{P}$ is the orthogonal projection on $\operatorname{ker}\left(L_{\text {maxwell }}\right)$ in $\mathcal{L}^{2}\left(\mathbb{R}^{3}, \mathbb{C}^{6}\right)$, as in Corollary 4.12 .

Theorem 4.10 can now be enhanced to yield

Theorem 4.14. The operator-valued function $R_{\text {maxwell }}(z)$ is well-defined (and analytic) for nonreal $z$ in the following functional setting.

$$
z \rightarrow \mathcal{P}^{\perp} R_{\text {maxwell }}(z) \in B\left(\mathcal{H}^{-1, s}\left(\mathbb{R}^{3}, \mathbb{C}^{6}\right), \mathcal{L}^{2,-l}\left(\mathbb{R}^{3}, \mathbb{C}^{6}\right)\right) .
$$

where $s, l>\frac{1}{2}$.

Furthermore, it can be extended continuously from $\mathbb{C}^{ \pm}$to $\mathbb{C}^{ \pm} \cup \mathbb{R}$, in this uniform operator topology. The limiting values (denoted again by $\mathcal{P}^{\perp} R_{\text {maxwell }}^{ \pm}(\lambda)$ ) are locally bounded and locally Hölder continuous in the same topology.

The extended function satisfies, for $z \in \mathbb{C}^{ \pm} \cup\{\mathbb{R} \backslash\{0\}\}$,

$$
\left(L_{\text {maxwell }}-z\right) \mathcal{P}^{\perp} R_{\text {maxwell }}(z) f=f-z \mathcal{P} R_{\text {maxwell }}(z) f, \quad f \in \mathcal{H}^{-1, s}\left(\mathbb{R}^{3}, \mathbb{C}^{6}\right),
$$$$
\text { where for } z=\lambda \in \mathbb{R} \backslash\{0\}, \quad \mathcal{P}^{\perp} R_{\text {maxwell }}(z)=\mathcal{P}^{\perp} R_{\text {maxwell }}^{ \pm}(\lambda) \text {. }
$$

Remark 4.15. Note that the operator $\mathcal{P} R_{\text {maxwell }}(z)$ is well-defined for $\operatorname{Imz} \neq 0$ and can therefore be extended continuously (in the sense of distributions) to the real axis, in view of the continuity of the left-hand side in Equation (4.41), as is established in the following proof.

Proof of the Proposition. The proof runs parallel to that of Proposition 4.5. In fact, in the functional setting of $B\left(\mathcal{H}^{-1, s}\left(\mathbb{R}^{3}, \mathbb{C}^{6}\right), \mathcal{H}^{-1,-l}\left(\mathbb{R}^{3}, \mathbb{C}^{6}\right)\right)$ the claims follow from the general theory, in view of Proposition 4.13. Also the proof of (4.41) is identical to that of (4.19).

Since the operator $L_{\text {maxwell }}$ is not elliptic, we only need to justify the stronger continuity claim (4.40), namely, the fact that $\mathcal{H}^{-1,-l}\left(\mathbb{R}^{3}, \mathbb{C}^{6}\right)$ can be replaced by $\mathcal{L}^{2,-l}\left(\mathbb{R}^{3}, \mathbb{C}^{6}\right)$ in the statement. However, the restriction of $L_{\text {maxwell }}$ to the subspace orthogonal to its kernel is elliptic, as seen from Equation (4.25). Therefore, for any $u$ in this subspace, the graph-norm $\|u\|_{-1,-l}+\left\|L_{\text {maxwell }} u\right\|_{-1,-l}$ is equivalent to the $\mathcal{L}^{2,-l}$ norm $\|u\|_{0,-l}$.

\section{STRONGLY PROPAGATIVE OR ISOTROPIC OPERATORS}

We now turn back to the study of the spectral structure of the general (constant coefficient) operator (1.2):

$$
L_{0}=L_{0, h o m}+M_{0}^{0}=\sum_{j=1}^{n} M_{j}^{0} D_{j}+M_{0}^{0} .
$$


Its $(K \times K$ matrix $)$ symbol is given by

$$
M_{0}(\xi)=M_{0, h o m}(\xi)+M_{0}^{0}=\sum_{j=1}^{n} M_{j}^{0} \xi_{j}+M_{0}^{0} .
$$

All the common physical systems (Dirac, Maxwell, wave propagation in elastic medium and others) share the basic property of being strongly propagative, according to the following definition.

Definition 5.1. [48]: The homogeneous operator $L_{0, h o m}=\sum_{j=1}^{n} M_{j}^{0} D_{j}$ is said to be strongly propagative if $M_{0, h o m}(\xi)$ has a kernel of fixed dimension $0 \leq d<K$, independent of $\xi=\left(\xi_{1}, \ldots, \xi_{n}\right) \in \mathbb{R}^{n} \backslash\{0\}$.

The nonzero eigenvalues of $M_{0, \text { hom }}$ have the following properties.

- They are positive-homogeneous of degree 1 .

- Let $Q_{\min }^{M_{0, h o m}}(\theta ; \xi), \theta \in \mathbb{C}$, be the minimal polynomial of $M_{0, h o m}(\xi)$. Let

$$
Z=\left\{\xi \in \mathbb{R}^{n} \backslash\{0\} / \text { the discriminant of } Q_{\min }^{M_{0, h o m}}(\theta ; \xi) \text { vanishes }\right\} \text {. }
$$

Then $\bar{Z}=Z \cup\{0\}$ is a closed cone of Lebesgue measure zero 48 .

- In $\mathbb{R}^{n} \backslash \bar{Z}$ every eigenvalue of $M_{0, h o m}(\xi)$ has constant multiplicity .

The distinct nonzero eigenvalues can therefore be enumerated as

$$
\mu_{\rho}(\xi)>\ldots>\mu_{1}(\xi)>0>\mu_{-1}(\xi)>\ldots>\mu_{-\rho}(\xi), \xi \in \mathbb{R}^{n} \backslash \bar{Z}
$$

The basic properties of these functions can be summarized as follows.

$$
\left\{\begin{array}{l}
(i) \mu_{k}(\xi) \text { is continuous on } \mathbb{R}^{n}, \text { and in fact real analytic on } \mathbb{R}^{n} \backslash \bar{Z} \\
(\text { ii }) \mu_{k}(\xi)=-\mu_{-k}(-\xi), k=1, \ldots, \rho, \xi \in \mathbb{R}^{n} \backslash \bar{Z} \\
(\text { iii }) \mu_{k}(\beta \xi)=\beta \mu_{k}(\xi), \beta>0, k=1, \ldots, \rho, \xi \in \mathbb{R}^{n} \backslash \bar{Z}
\end{array}\right.
$$

The corresponding projections are denoted by

$$
\left\{P_{k}(\xi) / \xi \in \mathbb{R}^{n} \backslash \bar{Z}\right\}_{0 \neq|k| \leq \rho} .
$$

Remark that for $\xi \in Z$ the disjointness property (5.3) is not valid, but the eigenvalues clearly retain the homogeneity property. In fact, they are all bounded on every sphere $|\xi|=r>0$, as is stated in the following claim.

Claim 5.2. There exist constants $c_{2}>c_{1}>0$ so that all nonzero eigenvalues satisfy

$$
c_{1} \leq\left|\mu_{ \pm k}\left(\frac{\xi}{|\xi|}\right)\right| \leq c_{2}, \quad \xi \neq 0, k=1, \ldots, \rho .
$$

Proof. The sphere $|\xi|=1$ is compact, so the boundedness of the nonzero eigenvalues follows from the "group continuity" [30, Section II.4] of these eigenvalues and the assumption that the operator is strongly propagative.

The inequality (5.6) follows from the homogeneity property.

Even though we do not treat in this paper the general nonhomogeneous strongly propagative case, we shall make here a comment concerning its possible eigenvalues. Suppose then that the constant matrix $M_{0}^{0} \neq 0$ is not a scalar matrix. 
Let us consider the possibility of having an eigenvalue $\eta$ of $M_{0}(\xi)$ that is independent of $\xi$ for $\xi$ in some open set $O \subseteq \mathbb{R}^{n}$. The existence of such an eigenvalue is equivalent to the fact that $\eta$ is an eigenvalue of $L_{0}$. In the homogeneous case $\left(M_{0}^{0}=0\right)$ we can only have $\eta=0$.

For $\eta$ to be such an eigenvalue we need

$$
\operatorname{det}\left(M_{0}(\xi)-\eta I_{K}\right)=0, \quad \xi \in O .
$$

Since the determinant is a polynomial in $\xi$, it follows that it actually vanishes for all $\xi \in \mathbb{R}^{n}$. In particular, $\eta$ is an eigenvalue of $M_{0}^{0}$.

We conclude that $\eta$ must be contained in the finite set (subset of the set of eigenvalues of $M_{0}^{0}$ )

$$
\Lambda=\text { the set of common eigenvalues of } M_{0}^{0}+\sum_{j=1}^{n} a_{j} M_{j}^{0}, \quad \forall a=\left(a_{1}, \ldots, a_{n}\right) \in \mathbb{R}^{n} \text {. }
$$

Any further spectral information, in our approach, requires a detailed study of the level surfaces of the eigenvalues (in analogy to the cases of the Dirac operator and the Maxwell system) . The information we need (in the general nonhomogeneous case) requires the use of delicate tools of real algebraic geometry and will not be attempted here. We remark that, to the best of our knowledge, the spectral study of this general class of operators (e.g., the Limiting Absorption Principle) has never been carried out.

A more restricted class is that of operators for which $Z=\emptyset$, as follows.

Definition 5.3. [47]: The operator $L_{0, \text { hom }}=\sum_{j=1}^{n} M_{j}^{0} D_{j}$ is said to be uniformly propagative if it is strongly propagative and, moreover, the eigenspace associated with every eigenvlaue has a constant dimension, independent of $\xi \in \mathbb{R}^{n} \backslash\{0\}$.

For simplicity in what follows we shall refer also to the associated symbols as "strongly" or "uniformly" propagative.

J. Rauch studied the asymptotic behavior of solutions of first-order hyperbolic systems, imposing the assumption of a uniformly propagative system [36, Assumption (1.3)]. Note that "the equations of electromagnetic and elastic waves in crystals are not uniformly propagative. However they are strongly propagative" 46, Introduction].

In our treatment we shall always assume that $L_{0, h o m}$ is strongly propagative.

We shall restrict our considerations to two classes of operators:

- Strongly propagative homogeneous operators, a generalization of the Maxwell system, as well as the massless Dirac operator.

- Nonhomogeneous isotropic operators (see Definition 5.15 below), a generalization of the massive Dirac operator.

\subsection{SPECTRAL STRUCTURE OF HOMOGENEOUS STRONGLY PROP-}

AGATIVE OPERATORS. The assumption that $M_{0}^{0}=0$ and $L_{0, \text { hom }}$ is strongly propagative permits an explicit representation of its domain as well as "partial coercivity" characterization, in full analogy to the Maxwell operator (see (4.23) ).

We define for every index $k= \pm 1, \ldots, k= \pm \rho$, the subspace

$$
X_{k}=\left\{f \in \mathcal{L}^{2}\left(\mathbb{R}^{n}, \mathbb{C}^{K}\right) / P_{k}(\xi) \widehat{f}(\xi)=\mu_{k}(\xi) \widehat{f}(\xi), \xi \in \mathbb{R}^{n} \backslash \bar{Z}\right\}
$$


where the projections $P_{k}(\xi)$ are as in (5.5).

These subspaces are clearly reducing for $L_{0, h o m}$. In view of (5.6) we obtain the (partial) coercivity property,

$$
\left|<M_{0, h o m}(\xi) \widehat{f}(\xi), \widehat{f}(\xi)>_{\mathbb{C}^{K}}\right| \geq c_{1}|\xi||\widehat{f}(\xi)|^{2}, \quad f \in X_{k}, 1 \leq|k| \leq \rho,
$$

(compare the analogous fact (4.24) for the Maxwell system). We therefore conclude that

$$
\operatorname{Dom}\left(L_{0, \text { hom }}\right)=\operatorname{ker}\left(L_{0, \text { hom }}\right) \oplus \sum_{1 \leq|k| \leq \rho} \oplus\left(X_{k} \cap \mathcal{H}^{1}\left(\mathbb{R}^{n}, \mathbb{C}^{K}\right)\right) .
$$

5.1.1. The Limiting Absorption Principle for homogeneous strongly propagative systems. Recall that the set $Z$ was defined in (5.2). Let $\mu_{j}(\xi), \xi \in$ $\mathbb{R}^{n} \backslash \bar{Z}, 0 \neq|j| \leq \rho$ be a nonzero eigenvalue. Let $\lambda \in \mathbb{R} \backslash\{0\}$ and consider the surface

$$
\Gamma_{j}(\lambda)=\left\{\xi \in \mathbb{R}^{n} \backslash \bar{Z} / \operatorname{sgn}(j)=\operatorname{sgn}(\lambda), \mu_{j}(\xi)=\lambda\right\} .
$$

It is an open smooth submanifold of codimension 1. It is bounded (and bounded away from the origin) in view of Claim 5.2 .

The homogeneity property implies that the surfaces are homothetic in the sense that

$$
\Gamma_{j}(\lambda)=\lambda \Gamma_{j}(1), \quad \lambda \neq 0 .
$$

The surface $\Gamma_{j}(1)$ plays a basic role in the wave propagation associated with the operator.

Definition 5.4. The surfaces $\Gamma_{j}(1)=\left\{\mu_{j}(\xi)=\operatorname{sgn}(j)\right\}$ are called the slowness surfaces of the system (see [47, Section 4]).

The term used in [17 is normal surfaces. We note that treatments by means of global Fourier integral operators necessitate a very careful study of these surfaces as well as very special assumptions on the system (see e.g. 31]).

Since $\Gamma_{j}(1)=-\Gamma_{-j}(1)$, we shall henceforth assume $j>0$, with $\lambda>0$.

The homogeneity of $\mu_{j}(\xi)$ implies, by the Euler identity, that

$$
<\xi, \nabla \mu_{j}(\xi)>_{\mathbb{R}^{n}}=\mu_{j}(\xi)=\lambda, \quad \xi \in \Gamma_{j}(\lambda),
$$

so that the Cauchy-Schwarz inequality and (5.6) yield

$$
\left|\nabla \mu_{j}(\xi)\right| \geq c_{1}>0, \quad \xi \in \Gamma_{j}(\lambda), \lambda>0,1 \leq j \leq \rho .
$$

Remark 5.5. The inequality (5.12) means that the "group velocity" at the wavefront $\Gamma_{j}$ is bounded away from zero. Compare with the analogous situation in the study of the asymptotic behavior of solutions of first-order systems [36. Equation $(1.7)]$.

Let $d \Sigma_{\Gamma_{j}(\lambda)}$ be the Lebesgue measure on $\Gamma_{j}(\lambda)$. The scaling property (5.11) yields

$$
d \Sigma_{\Gamma_{j}(\lambda)}=\lambda^{n-1} d \Sigma_{\Gamma_{j}(1)} .
$$

Let $\omega \in \Gamma_{j}(1)$ be a general point, with a corresponding $\lambda \omega \in \Gamma_{j}(\lambda)$. By (5.13) the traces of any bounded continuous function $f$ on the two submanifolds satisfy

$$
\int_{\Gamma_{j}(\lambda)}|f(\lambda \omega)|^{2} d \Sigma_{\Gamma_{j}(\lambda)}=\int_{\Gamma_{j}(1)} \lambda^{n-1}|f(\lambda \omega)|^{2} d \Sigma_{\Gamma_{j}(1)} .
$$


Define (using appropriate scaling) the trace maps of the Sobolev space $\mathcal{H}^{\theta}\left(\mathbb{R}^{n}\right)$ into $L^{2}\left(\Gamma_{j}(1)\right)$, by

$$
\left(\Phi_{\lambda}^{j} h\right)(\omega)=\lambda^{\frac{n-1}{2}} h(\lambda \omega), \quad \omega \in \Gamma_{j}(1), 1 \leq j \leq \rho .
$$

To estimate these trace maps we invoke [6, Lemma A.5]. The uniform lower bound (5.12) implies that the essential condition in that lemma $(|\nabla g| \leq d)$ is satisfied uniformly for any compact $K \Subset \Gamma_{j}(1)$. We conclude (by exhaustion) that the estimate can be applied to the smooth manifold $\Gamma_{j}(1)$, hence these maps are uniformly bounded for any $\theta>\frac{1}{2}$ :

$$
\sup _{\lambda>0}\left\{\left\|\Phi_{\lambda}^{j}\right\|_{B\left(\mathcal{H}^{\theta}\left(\mathbb{R}^{n}\right), \mathcal{L}^{2}\left(\Gamma_{j}(1)\right)\right)}\right\}<\infty
$$

and the operator-valued map $\lambda \hookrightarrow B\left(\mathcal{H}^{\theta}\left(\mathbb{R}^{n}\right), \mathcal{L}^{2}\left(\Gamma_{j}(1)\right)\right)$ is locally hölder continuous in the uniform operator topology (compare Lemma 3.1).

Let $\left\{E_{0, h o m}(\lambda), \lambda \in \mathbb{R}\right\}$ be the spectral family of $L_{0, h o m}$. Since $E_{0, h o m}(\lambda)$ commutes with $L_{0, h o m}$, it also has a symbol, which we denote by $E_{0, h o m}(\lambda ; \xi)$. The following claim gives an explicit expression in terms of the projections on the eigenspaces (assuming $\lambda>0$, with a similar expression for $\lambda<0$ ). We use $\chi_{B}$ as the indicator function for a set $B \subseteq \mathbb{R}^{n}$, namely, $\chi_{B}(x)=1\left(\operatorname{resp} . \chi_{B}(x)=0\right)$ if $x \in B$ (resp. $x \notin B$ ).

Claim 5.6. Let $\lambda>0$. Then,

$$
E_{0, h o m}(\lambda ; \xi)=\sum_{j=1}^{\rho} P_{-j}(\xi)+P_{0}(\xi)+\sum_{j=1}^{\rho} P_{j}(\xi) \chi_{\left\{\mu_{j}(\xi) \leq \lambda\right\}} .
$$

(refer to Equations (5.4) and (5.5) for notation of eigenvalues and projections).

If $\widehat{f}, \widehat{g} \in C_{0}^{\infty}\left(\mathbb{R}^{n} \backslash \bar{Z}\right)$ then, assuming $\lambda>0$, we obtain by a well known formula ("coarea formula" 24, Appendix C.3]) for differentiation of volume integrals

$$
\frac{d}{d \lambda}\left(E_{0, h o m}(\lambda) f, g\right)=\sum_{j=1}^{\rho} \int_{\Gamma_{j}(\lambda)} \frac{\left\langle P_{j}(\xi) \widehat{f}(\xi), P_{j}(\xi) \widehat{g}(\xi)>_{\mathbb{C}^{K}}\right.}{\left|\nabla \mu_{j}(\xi)\right|} d \Sigma_{\Gamma_{j}(\lambda)},
$$

where $d \Sigma_{\Gamma_{j}(\lambda)}$ is the Lebesgue surface measure (compare Equation (4.27)).

Note that the coarea formula requires global Lipschitz condition on $\mu_{j}(\xi)$. However, it is obtained by multiplying $\mu_{j}(\xi)$ by a cutoff smooth function such that $\varphi(\xi)=1$ on the supports of $\widehat{f}(\xi), \widehat{g}(\xi)$ and vanishes in a neighborhood of $\bar{Z}$. Then we see from (5.17) that for $\lambda>0$

$$
\frac{d}{d \lambda}\left(E_{0, h o m}(\lambda) f, g\right)=\sum_{j=1}^{\rho} \frac{d}{d \lambda} \int_{\mathbb{R}^{n}}<P_{j}(\xi) \chi_{\left\{\varphi(\xi) \mu_{j}(\xi) \leq \lambda\right\}} \widehat{f}(\xi), P_{j}(\xi) \widehat{g}(\xi)>_{\mathbb{C}^{K}} d \xi .
$$

Since the level set $\left\{\xi \mid \varphi(\xi) \mu_{j}(\xi)=\lambda\right\}$ is a smooth $(n-1)$-dimensional hypersurface for $\lambda>0$, one can apply the coarea formula to the right hand side of (5.19), obtaining (5.18).

The real analyticity of the functions $\mu_{j}(\xi), \xi \in \mathbb{R}^{n} \backslash \bar{Z}$, guarantees that Equation (5.18) can be repeatedly differentiated, using higher derivatives of $\widehat{f}(\xi), \widehat{g}(\xi)$.

We introduce the subspace of functions permitting such recurrent differentiation in the following definition. 
Definition 5.7. Let $\widehat{\Upsilon_{Z}^{s}}$ be the closure of $C_{0}^{\infty}\left(\mathbb{R}^{n} \backslash \bar{Z}, \mathbb{C}^{K}\right)$ in $\mathcal{H}^{s}\left(\mathbb{R}^{n}, \mathbb{C}^{K}\right)$, for $s>\frac{1}{2}$, and let $\Upsilon_{Z}^{s} \subseteq \mathcal{L}^{2, s}\left(\mathbb{R}^{n}, \mathbb{C}^{K}\right)$ be the subspace of its inverse Fourier transforms. It is a closed subspace, equipped with the same norm $\|\cdot\|_{0, s}$ (3.1).

In the following claim we characterize the orthogonal complement of $\Upsilon_{Z}^{s}$.

Claim 5.8. Let $\left(\Upsilon_{Z}^{s}\right)^{\perp} \subseteq \mathcal{L}^{2, s}\left(\mathbb{R}^{n}, \mathbb{C}^{K}\right), s>\frac{1}{2}$, be the orthogonal complement to $\Upsilon_{Z}^{s}$ (using the scalar product associated with (3.1)). Let $h(x) \in\left(\Upsilon_{Z}^{s}\right)^{\perp}$. Then the Fourier transform of $\left(1+|x|^{2}\right)^{s} h(x)$ is supported on $\bar{Z}$ :

$$
\text { supp } \mathcal{F}\left\{\left(1+|x|^{2}\right)^{s} h(x)\right\}(\xi) \subseteq \bar{Z} \text {. }
$$

Remark 5.9. Note that if $h(x) \in \mathcal{L}^{2, s}\left(\mathbb{R}^{n}, \mathbb{C}^{K}\right)$ then $\left(1+|x|^{2}\right)^{\frac{s}{2}} h(x) \in \mathcal{L}^{2}\left(\mathbb{R}^{n}, \mathbb{C}^{K}\right)$ and $\left(1+|x|^{2}\right)^{s} h(x) \in \mathcal{L}^{2,-s}\left(\mathbb{R}^{n}, \mathbb{C}^{K}\right)$.

Proof of Claim: Let $\psi \in \mathcal{L}^{2, s}\left(\mathbb{R}^{n}, \mathbb{C}^{K}\right)$ such that $\widehat{\psi} \in C_{0}^{\infty}\left(\mathbb{R}^{n} \backslash \bar{Z}, \mathbb{C}^{K}\right)$. The scalar product in $\mathcal{L}^{2, s}\left(\mathbb{R}^{n}, \mathbb{C}^{K}\right)$ can be expressed as

$$
(h, \psi)_{0, s}=\int_{\mathbb{R}^{n}}<\widehat{h}(\xi),(I-\Delta)^{s} \widehat{\psi}(\xi)>_{\mathbb{C}^{K}} d \xi=<(I-\Delta)^{s} \widehat{h}, \widehat{\psi}>,
$$

where $<,>$ in the last term stands for the $\mathcal{H}^{-s}\left(\mathbb{R}^{n}, \mathbb{C}^{K}\right), \mathcal{H}^{s}\left(\mathbb{R}^{n}, \mathbb{C}^{K}\right)$ pairing. The assumption $h(x) \in\left(\Upsilon_{Z}^{s}\right)^{\perp}$ means that $(h, \psi)_{0, s}=0$ hence

$$
\operatorname{supp}(I-\Delta)^{s} \widehat{h}=\operatorname{supp} \mathcal{F}\left\{\left(1+|x|^{2}\right)^{s} h(x)\right\} \subseteq \bar{Z} .
$$

Remark 5.10. Since $Z$ has Lebesgue measure zero (in $\mathbb{R}^{n}$ ) it is clear that $\Upsilon_{Z}^{s}$ is dense in $\mathcal{L}^{2}\left(\mathbb{R}^{n}, \mathbb{C}^{K}\right)$. However, if $Z$ is "large" in some " $(n-1)$ - dimensional" sense, then $\Upsilon_{Z}^{s}$ is not necessarily equal to $\mathcal{L}^{2, s}\left(\mathbb{R}^{n}, \mathbb{C}^{K}\right)$. In fact, using the terminology of [28, $\Upsilon_{Z}^{s}$ is equal to $\mathcal{L}^{2, s}\left(\mathbb{R}^{n}, \mathbb{C}^{K}\right)$ only if $Z$ is "s-polar". In other words, if it has zero Bessel potential theoretic capacity of order $s$ [32, Sections 10.4, 13.2].

Given the special algebraic structure of $Z$ (see (5.2) ) we introduce the following conjecture.

CONJECTURE 5.11. The set $Z$ is $s$-polar for $s \in\left(\frac{1}{2}, \frac{3}{2}\right)$, hence the subspace $\Upsilon_{Z}^{s}$ is equal to $\mathcal{L}^{2, s}\left(\mathbb{R}^{n}, \mathbb{C}^{K}\right)$.

In conjunction with (5.12) and (5.16) we conclude from Equation (5.18) that, for any $s>\frac{1}{2}$ there exists a constant $C>0$, depending only on $s, c_{1}$, so that, for all $\lambda>0$, and all $f, g \in \Upsilon_{Z}^{s}$,

$$
\begin{aligned}
& \left|\frac{d}{d \lambda}\left(E_{0, h o m}(\lambda) f, g\right)\right| \leq \frac{1}{c_{1}} \sum_{j=1}^{\rho} \int_{\Gamma_{j}(\lambda)}|\widehat{f}(\xi)| \cdot|\widehat{g}(\xi)| d \Sigma_{\Gamma_{j}(\lambda)} \\
= & \frac{1}{c_{1}} \sum_{j=1}^{\rho} \int_{\Gamma_{j}(1)}\left|\Phi_{\lambda}^{j} \widehat{f}(\omega)\right|\left|\Phi_{\lambda}^{j} \widehat{g}(\omega)\right| d \Sigma_{\Gamma_{j}(1)} \leq C\|f\|_{0, s}\|g\|_{0, s} .
\end{aligned}
$$

Any continuous functional on the closed subspace $\Upsilon_{Z}^{s}$ can be uniquely extended to a functional on $\mathcal{L}^{2, s}\left(\mathbb{R}^{n}, \mathbb{C}^{K}\right.$ ) (namely, a function in $\mathcal{L}^{2,-s}\left(\mathbb{R}^{n}, \mathbb{C}^{K}\right)$ ) by defining it as zero on the orthogonal complement.

It follows (compare Proposition 4.8) that 
Corollary 5.12. There exists a map

$$
\widetilde{A_{0, h o m}}(\lambda) \in B\left(\Upsilon_{Z}^{s}, \mathcal{L}^{2,-s}\left(\mathbb{R}^{n}, \mathbb{C}^{K}\right)\right), s>\frac{1}{2},
$$

so that

$$
\frac{d}{d \lambda}\left(E_{0, h o m}(\lambda) f, f\right)=<\widetilde{A_{0, h o m}}(\lambda) f, f>, \quad f \in \Upsilon_{Z}^{s},
$$

where $<,>$ is the $\left(\mathcal{L}^{2,-s}\left(\mathbb{R}^{n}, \mathbb{C}^{K}\right), \mathcal{L}^{2, s}\left(\mathbb{R}^{n}, \mathbb{C}^{K}\right)\right)$ pairing.

The map $\widetilde{A_{0, h o m}}(\lambda)$ is uniformly bounded

$$
\left\|\widetilde{A_{0, h o m}}(\lambda)\right\|_{B\left(\Upsilon_{Z}^{s}, \mathcal{L}^{2,-s}\left(\mathbb{R}^{n}, \mathbb{C}^{K}\right)\right)} \leq C, \quad \lambda \neq 0,
$$

and locally Hölder continuous in the uniform operator topology.

The global uniform boundedness (5.24) will play a crucial role in the spacetime estimates of Section 8 .

The general theory (see Theorem6.2 below with $\mathcal{X}=\Upsilon_{Z}^{s}$ ) now yields the LAP in this case as follows.

Theorem 5.13. Let $R_{0, h o m}(z)=\left(L_{0, h o m}-z\right)^{-1}$, Im $z \neq 0$. For any $s>\frac{1}{2}$ the limits

$$
R_{0, h o m}^{ \pm}(\mu)=\lim _{\varepsilon \downarrow 0} R_{0, h o m}(\mu \pm i \varepsilon), \mu \in \mathbb{R} \backslash\{0\},
$$

exist in the uniform operator topology of $B\left(\Upsilon_{Z}^{s}, \mathcal{L}^{2,-s}\left(\mathbb{R}^{n}, \mathbb{C}^{K}\right)\right)$.

Furthermore, the limit functions $R_{0, \text { hom }}^{ \pm}(\mu), \mu \in \mathbb{R} \backslash\{0\}$, are locally bounded and locally Hölder continuous with respect to the uniform operator topology.

Remark 5.14. The LAP result of Theorem 5.13 was proved by Weder in 45, 46] for intervals interior to $\mathbb{R} \backslash\{0\}$, in the operator space $B\left(\mathcal{L}^{2, s}\left(\mathbb{R}^{n}, \mathbb{C}^{K}\right), \mathcal{H}^{1,-s}\left(\mathbb{R}^{n}, \mathbb{C}^{K}\right)\right)$. We emphasize that we do not believe that the presence of the singular set $Z$ could be entirely dismissed. The proof in [45] relies on the commutator approach, and we were not quite able to follow the details there. On the other hand the proof in [46] is essentially based on the methodology of trace maps. It seems to us to be fundamentally flawed, and this impression has not changed even after a long correspondence with him. The proof of [46. Theorem A.1] involves a deformation map of the slowness surface $\Gamma_{j}(1)$ onto the unit sphere followed by an application of the trace theorem on the sphere. Thus, the measure $d w_{j}$ on $\Gamma_{j}(1)$ is defined by the radial projection on the unit sphere (see [46. Eq. (A.20)]) so as to obtain a "polar decomposition" $d^{n} k=\rho^{n-1} d \rho d w_{j}$ [46, Eq. (A.19)]. This is of course wrong, since the coarea formula is ignored. That formula introduces a denominator $\left|\nabla \mu_{j}(k)\right|$ (see Eq. (5.18)) in the last expression, which is singular on Z. Effectively, he argues that $Z$ is of "measure zero" in $\Gamma_{j}(1)$, so our $\Upsilon_{Z}^{s}$ can be identified with $\left.\mathcal{L}^{2, s}\left(\mathbb{R}^{n}, \mathbb{C}^{K}\right)\right)$. This whole argument is applicable in obtaining a trace on every starshaped surface, no matter how singular, and this is clearly wrong, see Theorem 2.3 in [1] and Remark 5.10.

5.2. SPECTRAL DENSITY OF ISOTROPIC OPERATORS. . The examples of the Dirac and Maxwell operators motivate our next definition. In fact, like these two examples, all physical models where there is no "built in" preference for specific (spatial) directions, naturally fall into the category of isotropic operators [47, Section 4], that we recall next. 
Definition 5.15. The operator $L_{0}(1.2)$ is said to be isotropic if the eigenvalues of its symbol (see (5.1) $) M_{0}(\xi)$ are functions of $|\xi|$.

We assume in addition that $L_{0, h o m}$ is strongly propagative (Definition 5.1).

If $L_{0}$ is homogeneous, namely, $M_{0}^{0} \equiv 0$, then $M_{0}(\xi)=M_{0, h o m}(\xi)$. By definition the zero eigenvalue $\mu_{0}(\xi)=0$ is of fixed dimension $d_{0} \geq 0$. The singular set $Z$ (5.2) is empty and the eigenvalues $\mu_{k}$ (see Equation (5.3) ) satisfy

$$
\mu_{k}(\xi)=\operatorname{sign}(k) \mu_{|k|}^{c}|\xi|, \quad \xi \neq 0, \pm k=1,2, \ldots, J,
$$

where $\mu_{k}^{c}$ are positive constants such that $\mu_{J}^{c}>\ldots>\mu_{1}^{c}>0$.

Each eigenvalue $\mu_{k}$ is of fixed dimension $d_{k}>0, \pm k=1,2, \ldots, J$, and $d_{k}=d_{-k}$. In particular, in this case the operator is uniformly propagative (Definition 5.3).

It is therefore a special case of the class considered above in Subsection 5.1 and does not require a further consideration here.

We now turn to the nonhomogeneous operator.

In this case the eigenvalues are functions of a single variable $r=|\xi|$, and we denote them by

$$
\lambda_{1}(r) \leq \lambda_{2}(r) \leq \cdots \leq \lambda_{\rho}(r) .
$$

However now these eigenvalues are not homogeneous functions of $r>0$, and, unlike the homogeneous case, their multiplicity is not fixed. In other words, two (or more) different eigenvalues $\lambda_{i}(r) \neq \lambda_{j}(r)$ can "coalesce" at a point $r=r_{0}$. Such a point $r_{0}$ is called " a crossing point".

As we shall see below, it will be necessary to forsake their ordering in order to maintain their analyticity.

Since $L_{0}$ is isotropic, the eigenvalues of

$$
M_{0}(\xi)=M_{0, h o m}(\xi)+M_{0}^{0}=|\xi| \sum_{j=1}^{n} M_{j}^{0} \omega_{j}+M_{0}^{0} \quad(\omega=\xi /|\xi|)
$$

are functions of $|\xi|$. In particular, the eigenvalues of $M_{0}(\xi)=M_{0}(|\xi| \omega)$, together with their multiplicities, are independent of $\omega \in \mathbb{S}^{n-1}$.

By virtue of this fact, we can take $\omega=e_{1}=(1,0, \cdots, 0)$, and study the eigenvalues of

$$
M_{0}\left(|\xi| e_{1}\right)=|\xi| M_{1}^{0}+M_{0}^{0} .
$$

Taking $r=|\xi|>0$, the eigenvalue study is reduced to the study of the symmetric matrix, depending (linearly) on a positive parameter,

$$
T(r)=r M_{1}^{0}+M_{0}^{0}, \quad r>0 .
$$

However, it is useful to regard $T(r)$ as a function of the coordinate $r \in \mathbb{R}$. We can now appeal directly to the analytic perturbation theory of Hermitian matrices 30 , Section II.6 ].

We conclude that the eigenvalues $\lambda_{1}(r), \cdots, \lambda_{\rho}(r)$ of $T(r)$ are analytic functions of $r \in \mathbb{R}$. The sum of their multiplicities is $K$, and each of them is constant in intervals not containing crossing points, as will be explained below.

We denote by

$$
P_{1}(\xi), \cdots, P_{\rho}(\xi),
$$

the corresponding projections. 
Note that $P_{j}(\xi)$ cannot be assumed to depend on $|\xi|$. Furthermore, in every closed interval $[\alpha, \beta] \Subset \mathbb{R}$ there is at most a finite number of crossing points. In fact, the fact that $T(r)$ depends linearly on $r$, enables us to claim even more.

Claim 5.16. There is at most a finite number of crossing points in the whole real line $r \in \mathbb{R}$.

Proof. The crossing points are zeros of the discriminant of the minimal polynomial of $T(r)$, as a function of $r \in \mathbb{R}$. However, clearly this discriminant is an algebraic function of $r$, and as such can have at most a finite number of zeros.

As already noted above, the analytic perturbation theory of Hermitian matrices [30, Section II.6 ] implies that $\lambda_{1}(r), \cdots, \quad \lambda_{\rho}(r)$ are analytic functions of $r>0$. Clearly, to maintain them as analytic functions we cannot order them, as there may be crossing points. We define the crossing values as the finite set

$$
\left\{\lambda \in \mathbb{R} / \lambda=\lambda_{j}(q) \text {, for some } j \text { and some crossing point } q\right\} .
$$

Remark 5.17. In the isotropic case the singular set $Z$ in Equation (15.2) can be expressed explicitly as follows.

$$
Z=\left\{\begin{array}{l}
\emptyset, \quad \text { the homogeneous case, } \\
\text { a finite union of spheres in the nonhomogeneous case. }
\end{array}\right.
$$

If a sphere of radius $r>0$ (centered at the origin) is included in $Z$, then $r$ is a crossing point.

Define the spherical surfaces

$$
\Gamma_{j}(\lambda)=\left\{\xi \in \mathbb{R}^{n} / \lambda_{j}(|\xi|)=\lambda\right\}, \lambda \in \mathbb{R} \backslash \Lambda .
$$

If $\Gamma_{j}(\lambda) \subseteq Z$ then $\lambda$ is a crossing value.

However, as noted above, even at crossing points the eigenvalues remain analytic. Thus, the only values of $\lambda$ to be excluded are the critical values, where, by definition, $\lambda=\lambda_{j}(r)$ and $\lambda_{j}^{\prime}(r)=0$ for some $j \in\{1,2, \ldots, \rho\}$ and some $r \in \mathbb{R}$.

Claim 5.18. There is at most a finite number of critical values of the eigenvalues $\lambda_{j}(r), j=1,2, \ldots, \rho$.

Proof. The functions $\lambda_{j}(r)$ are roots of the algebraic equation $\operatorname{det}\left(T(r)-\lambda I_{K}\right)=0$ $(T(r)$ is defined in (5.29) $)$, and are therefore algebraic functions of the real variable $r \in \mathbb{R}$. We can now apply the classical argument in [27, Section 14.3]; the set of critical values is of measure zero by Sard's theorem, hence being semi-algebraic set it must be finite .

Recall the definition (5.7) of the finite set $\Lambda$, that contains all possible eigenvalues of $L_{0}$.

Definition 5.19. The set $\Lambda$ of (5.7) is extended (retaining the same notation) to include also the finitely many critical values.

Remark 5.20. Continuing Remark 5.5; the requirement that $\lambda$ is not a critical value means that the "group velocity" at the wavefront $\Gamma_{j}(\lambda)$ is bounded away from zero, for all $j \in\{1,2, \ldots, \rho\}$.

Thus, the only values of the spectral parameter to be avoided are the values in $\Lambda$, and not necessarily all crossing values. Let $\lambda \in \mathbb{R} \backslash \Lambda$, 
5.2.1. The Limiting Absorption Principle for isotropic operators. Let $E_{0}(\lambda)$ be spectral family associated with $L_{0}$. Since $E_{0}(\lambda)$ commutes with $L_{0}$, it also has a symbol, which we denote by $E_{0}(\lambda ; \xi)$. As in the case of Claim 5.6 we have here.

Claim 5.21. Let $\lambda \in \mathbb{R} \backslash \Lambda$. Then, with projections $P_{j}$ as in (5.30),

$$
E_{0}(\lambda ; \xi)=\sum_{j=1}^{\rho} P_{j}(\xi) \chi_{\left\{\lambda_{j}(|\xi|) \leq \lambda\right\}}
$$

Corollary 5.22. If $\hat{f}, \hat{g} \in C_{0}^{\infty}\left(\mathbb{R}^{n}, \mathbb{C}^{K}\right)$ and $\lambda \in \mathbb{R} \backslash \Lambda$, then

$$
\begin{aligned}
\frac{d}{d \lambda} & \left(E_{0}(\lambda) f, g\right)=\sum_{j=1}^{\rho} \int_{\Gamma_{j}(\lambda)} \frac{<P_{j}(\xi) \hat{f}(\xi), \hat{g}(\xi)>_{\mathbb{C}^{K}}}{\left|\nabla \lambda_{j}(\xi)\right|} \chi_{\left\{\lambda_{j}(|\xi|)=\lambda\right\}} d \Sigma_{r_{j}} \\
& =\sum_{j=1}^{\rho}\left|\lambda_{j}^{\prime}\left(r_{j}\right)\right|^{-1} \int_{\Gamma_{j}(\lambda)}<P_{j}(\xi) \hat{f}(\xi), \hat{g}(\xi)>_{\mathbb{C}^{K}} \chi_{\left\{\lambda_{j}(|\xi|)=\lambda\right\}} d \Sigma_{r_{j}} .
\end{aligned}
$$

with $d \Sigma_{r_{j}}$ being the Lebesgue surface measure on the sphere $\Gamma_{j}(\lambda)$ of radius $r_{j}>0$ such that $\lambda_{j}\left(r_{j}\right)=\lambda$ (see (5.31) $)$.

Remark 5.23. In Equation (5.33) we know that $\lambda_{j}^{\prime}\left(r_{j}\right) \neq 0$ since $\lambda$ is not a critical value.

In analogy to the case of the strongly propagative system (Theorem 5.13) we can state here the LAP as following from the general theory.

Theorem 5.24. Let $L_{0}(D)$ be isotropic and $R_{0}(z)=\left(L_{0}-z\right)^{-1}$, Im $z \neq 0$. Then for any $s>\frac{1}{2}$ the limits

$$
R_{0}^{ \pm}(\mu)=\lim _{\varepsilon \downarrow 0} R_{0}(\mu \pm i \varepsilon), \mu \in \mathbb{R} \backslash \Lambda,
$$

exist in the uniform operator topology of $B\left(\mathcal{L}^{2, s}\left(\mathbb{R}^{n}, \mathbb{C}^{K}\right), \mathcal{L}^{2,-s}\left(\mathbb{R}^{n}, \mathbb{C}^{K}\right)\right)$.

Furthermore, the limit functions $R_{0}^{ \pm}(\mu), \mu \in \mathbb{R} \backslash \Lambda$, are locally bounded and locally Hölder continuous with respect to the uniform operator topology.

\section{THE CLASS OF PERTURBED OPERATORS}

We now turn to the study of the spectral structure of perturbations of the operators introduced in Section 2, Our ultimate goal is the study of the operator $L$ introduced in (1.1). This is done by regarding this operator as a perturbation of $L_{0}$, as given in (1.2).

We take the most basic perturbation of the form

$$
L=L_{0}+V(x)
$$

where $V(x)$ is a Hermitian $K \times K$ matrix that decays as $|x| \rightarrow \infty$. In Subsection 6.1 we expound the abstract theory of such perturbations. In particular, general conditions are given that imply the absolute continuity (and LAP) of the continuous spectrum, apart possibly from a discrete sequence of embedded eigenvalues.

In subsection 6.2 we apply the general theory to the potential perturbation of the Dirac operator. Observe that this includes the case of the magnetic Dirac operator, as the magnetic field can be merged into the potential perturbation. We choose to deal with this example in detail as it illustrates the applicability of the general theory, and also allows us to give decay conditions on the potential, to the effect 
that there are only finitely many eigenvalues in the spectral gap (of the massive Dirac operator, see Subsubsection 6.2.1.

The perturbed Maxwell system can also be reduced to the case of potential perturbation [42, Section 1.4], but we choose not to treat it here in detail, as the paper is already quite long.

\subsection{GENERAL THEORY: PERTURBATION BY A POTENTIAL. .}

Our treatment is based on the general theory expounded in [7, Sections 3-4]. The abstract setting will allow us to consider various operator settings in a unified way. We briefly recall some definitions and statements that will be needed here.

Let $H$ be a self-adjoint operator in $\mathcal{L}^{2}\left(\mathbb{R}^{n}, \mathbb{C}^{K}\right)$. The scalar product in $\mathcal{L}^{2}\left(\mathbb{R}^{n}, \mathbb{C}^{K}\right)$ is denoted by $($,$) .$

Suppose that there exists a Hilbert space such that $\mathcal{X} \subseteq \mathcal{L}^{2}\left(\mathbb{R}^{n}, \mathbb{C}^{K}\right)$, and the embedding is dense and continuous. In other words, $\mathcal{X}$ can be considered as a dense subspace of $\mathcal{L}^{2}\left(\mathbb{R}^{n}, \mathbb{C}^{K}\right)$, equipped with a stronger norm. Then, of course, $\mathcal{X} \hookrightarrow \mathcal{L}^{2}\left(\mathbb{R}^{n}, \mathbb{C}^{K}\right) \hookrightarrow \mathcal{X}^{*}$, where $\mathcal{X}^{*}$ is the anti-dual of $\mathcal{X}$; the continuous additive functionals $l$ on $\mathcal{X}$, such that $l(\alpha v)=\bar{\alpha} l(v), \alpha \in \mathbb{C}$.

We use $\|x\|_{\mathcal{X}},\left\|x^{*}\right\|_{\mathcal{X}^{*}}$ for the norms in $\mathcal{X}, \mathcal{X}^{*}$, respectively, and designate by $<,>$ the $\left(\mathcal{X}^{*}, \mathcal{X}\right)$ pairing.

The (linear) embedding $h \in \mathcal{L}^{2}\left(\mathbb{R}^{n}, \mathbb{C}^{K}\right) \hookrightarrow h^{*} \in \mathcal{X}^{*}$ is obtained as usual by the scalar product $\left(\right.$ in $\left.\mathcal{L}^{2}\left(\mathbb{R}^{n}, \mathbb{C}^{K}\right)\right), h^{*}(x)=(h, x)$.

We introduce still another Hilbert space $\mathcal{X}_{H}^{*}$, which is a dense subspace of $\mathcal{X}^{*}$, equipped with a stronger norm (so that the embedding $\mathcal{X}_{H}^{*} \hookrightarrow \mathcal{X}^{*}$ is continuous). However, we do not require that $\mathcal{L}^{2}\left(\mathbb{R}^{n}, \mathbb{C}^{K}\right)$ be embedded in $\mathcal{X}_{H}^{*}$. As indicated by the notation, $\mathcal{X}_{H}^{*}$ may depend on $H$. A typical case would be when $H$ can be extended as a densely defined operator in $\mathcal{X}^{*}$ and $\mathcal{X}_{H}^{*}$ would be its domain there, equipped with the graph norm.

Let $\{E(\lambda)\}$ be the spectral family of $H$. We denote by $E(B)$ the spectral projection on any Borel set $B$ (so that $E(\lambda)=E(-\infty, \lambda))$.

Definition 6.1. Let $U \subseteq \mathbb{R}$ be open and let $0<\alpha \leq 1$. Assume that $U$ is of full spectral measure, namely, $E(\mathbb{R} \backslash U)=0$. Then $H$ is said to be of type $\left(\mathcal{X}, \mathcal{X}_{H}^{*}, \alpha, U\right)$ if the following conditions are satisfied:

(1) The operator-valued function

$$
\lambda \rightarrow E(\lambda) \in B\left(\mathcal{X}, \mathcal{X}^{*}\right), \quad \lambda \in U,
$$

is weakly differentiable with a locally Hölder continuous derivative in $B\left(\mathcal{X}, \mathcal{X}_{H}^{*}\right)$; that is, there exists an operator-valued function

$$
\lambda \rightarrow A(\lambda) \in B\left(\mathcal{X}, \mathcal{X}_{H}^{*}\right), \quad \lambda \in U,
$$

so that

$$
\frac{d}{d \lambda}(E(\lambda) x, y)=<A(\lambda) x, y>, \quad x, y \in \mathcal{X}, \lambda \in U,
$$

and such that for every compact interval $K \subseteq U$, there exists an $M_{K}>0$ satisfying

$$
\|A(\lambda)-A(\mu)\|_{B\left(\mathcal{X}, \mathcal{X}_{H}^{*}\right)} \leq M_{K}|\lambda-\mu|^{\alpha}, \quad \lambda, \mu \in K .
$$


(2) For every bounded open set $J \subseteq U$ and for every compact interval $K \subseteq J$, the operator-valued function (defined in the weak sense)

$$
z \rightarrow \int_{U \backslash J} \frac{A(\lambda)}{\lambda-z} d \lambda, \quad z \in \mathbb{C}, \Re z \in K,|\Im z| \leq 1,
$$

takes values and is Hölder continuous in the uniform operator topology of $B\left(\mathcal{X}, \mathcal{X}_{H}^{*}\right)$, with exponent $\alpha$.

We can now state the basic theorem, concerning the Limiting Absorption Principle (LAP) in this setting. We use the notation $\mathbb{C}^{ \pm}=\{z \in \mathbb{C}, \pm \Im z>0\}$, and denote by $R(z)=(H-z)^{-1}, z \in \mathbb{C}^{ \pm}$, the resolvent of $H$.

Theorem 6.2. Let $H$ be of type $\left(\mathcal{X}, \mathcal{X}_{H}^{*}, \alpha, U\right)$ (where $U \subseteq \mathbb{R}$ is open and $0<\alpha \leq$ 1). Then $H$ satisfies the LAP in $U$. More explicitly, the limits

$$
R^{ \pm}(\lambda)=\lim _{\varepsilon \downarrow 0} R(\lambda \pm i \varepsilon), \quad \lambda \in U,
$$

exist in the uniform operator topology of $B\left(\mathcal{X}, \mathcal{X}_{H}^{*}\right)$ and the extended operator-valued function

$$
R(z)= \begin{cases}R(z), & z \in \mathbb{C}^{+}, \\ R^{+}(z), & z \in U\end{cases}
$$

is locally Hölder continuous in the same topology (with exponent $\alpha$ ).

A similar statement applies when $\mathbb{C}^{+}$is replaced by $\mathbb{C}^{-}$, but note that the limiting values $R^{ \pm}(\lambda)$ are in general different.

Remark 6.3. In view of the Stieltjes formula we have

$$
A(\lambda)=\frac{1}{2 \pi i}\left(R^{+}(\lambda)-R^{-}(\lambda)\right), \quad \lambda \in U .
$$

In particular, $H$ is absolutely continuous in $U$.

We now consider a perturbation by a potential function $V(x)$. To deal with the requirements on $V$ in this framework we introduce the following definition.

Definition 6.4. An operator $V: \mathcal{X}_{H}^{*} \rightarrow \mathcal{X}$ will be called

(1) Short-range with respect to $H$ if it is compact.

(2) Symmetric if $D(H) \cap \mathcal{X}_{H}^{*}$ is dense in $\mathcal{L}^{2}\left(\mathbb{R}^{n}, \mathbb{C}^{K}\right)$ and the restriction of $V$ to $D(H) \cap \mathcal{X}_{H}^{*}$ is symmetric in $\mathcal{L}^{2}\left(\mathbb{R}^{n}, \mathbb{C}^{K}\right)$.

The following lemma shows that (with some additional assumption) the operator $H+V$ is well defined.

Lemma 6.5. Let $H$ be of type $\left(\mathcal{X}, \mathcal{X}_{H}^{*}, \alpha, U\right)$ and let $V$ be short-range and symmetric. Suppose that there exists $z \in \mathbb{C}, \Im z \neq 0$, and a linear subspace $D_{z} \subseteq D(H) \cap \mathcal{X}_{H}^{*}$ such that $(H-z)\left(D_{z}\right)$, the image of $D_{z}$ under $H-z$, is dense in $\mathcal{X}$.

Then $P=H+V$, defined on $D(H) \cap \mathcal{X}_{H}^{*}$, is essentially self-adjoint.

In what follows we always assume that $H$ is of type $\left(\mathcal{X}, \mathcal{X}_{H}^{*}, \alpha, U\right)$ and that $V$ is short-range and symmetric. Thus, by the lemma, $P=H+V$ can be extended as a self-adjoint operator with domain $D(P) \supseteq D(H) \cap \mathcal{X}_{H}^{*}$, and we retain the notation $P$ for this extension.

Our aim is to study the spectral properties of $P$, particularly the LAP, in this abstract framework. 
Denote by $S(z)=(P-z)^{-1}, \Im z \neq 0$, the resolvent of $P$. Our starting point is the resolvent equation

$$
S(z)(I+V R(z))=R(z), \quad \Im z \neq 0 .
$$

It can be shown that the inverse $(I+V R(z))^{-1}$ exists on $\mathcal{X}$ if $\Im z \neq 0$. This leads to

$$
S(z)=R(z)(I+V R(z))^{-1}
$$

where the equality is certainly valid from $\mathcal{X} \rightarrow \mathcal{X}_{H}^{*}$.

Suppose now that $\lambda \in U$. In view of Theorem 6.2 and the assumption on $V$ we have

$$
\lim _{\varepsilon \downarrow 0} V R(\lambda \pm i \varepsilon)=V R^{ \pm}(\lambda) \text { in } B(\mathcal{X}) .
$$

Thus, if $\left(I+V R^{ \pm}(\lambda)\right)^{-1}$ exists (in $B(\mathcal{X})$ ), then Eq. (6.3) implies the existence of the limits

$$
S^{ \pm}(\lambda)=\lim _{\varepsilon \downarrow 0} S(\lambda \pm i \varepsilon)=R^{ \pm}(\lambda)\left(I+V R^{ \pm}(\lambda)\right)^{-1},
$$

in the uniform operator topology of $B\left(\mathcal{X}, \mathcal{X}_{H}^{*}\right)$.

Let $\lambda \in U$ be a point at which, say, $\left(I+V R^{+}(\lambda)\right)^{-1}$ does not exist (in $\left.B(\mathcal{X})\right)$. Since $V R^{+}(\lambda)$ is compact in $\mathcal{X}$, there exists a non-zero $\phi \in \mathcal{X}$ so that

$$
\phi=-V R^{+}(\lambda) \phi
$$

Let $\psi=R^{+}(\lambda) \phi \in \mathcal{X}_{H}^{*}$. Then

$$
<\psi, \phi>=-\lim _{\varepsilon \downarrow 0}(R(\lambda+i \varepsilon) \phi, V R(\lambda+i \varepsilon) \phi) .
$$

By the symmetry of $V$ the right-hand side of this equality is real, so we conclude that $\Im<R^{+}(\lambda) \phi, \phi>=0$, and invoking Eq. (6.2) we conclude that

$$
<A(\lambda) \phi, \phi>=0 \text {. }
$$

Now the form $\langle A(\lambda) x, y\rangle=\frac{d}{d \lambda}(E(\lambda) x, y)$ on $\mathcal{X} \times \mathcal{X}$ is symmetric and positive semi-definite. Hence, for every $y \in \mathcal{X}$,

$$
|<A(\lambda) \phi, y>| \leq<A(\lambda) \phi, \phi>^{\frac{1}{2}}<A(\lambda) y, y>^{\frac{1}{2}}=0,
$$

and we conclude that

$$
A(\lambda) \phi=0
$$

In particular, $R^{+}(\lambda) \phi=R^{-}(\lambda) \phi$ and

$$
\phi=-V R^{ \pm}(\lambda) \phi
$$

Definition 6.6. We designate by $\Sigma_{P}$ the set

$\Sigma_{P}=\left\{\lambda \in U /\right.$ There exists a non-zero $\phi_{\lambda} \in \mathcal{X}$ such that $\left.\phi_{\lambda}=-V R^{ \pm}(\lambda) \phi_{\lambda}\right\}$.

Remark 6.7. The set $\Sigma_{P}$ is (relatively) closed in $U$. Indeed, if $\left(I+V R^{ \pm}\left(\lambda_{0}\right)\right)^{-1}$ exists (in $B(\mathcal{X}))$, then $\left(I+V R^{ \pm}(\lambda)\right)^{-1}$ exists for $\lambda$ close to $\lambda_{0}$.

The discussion above leads to the following theorem.

Theorem 6.8. The operator $P=H+V$ satisfies the LAP in $U \backslash \Sigma_{P}$, in the uniform operator topology of $B\left(\mathcal{X}, \mathcal{X}_{H}^{*}\right)$, and the limiting values of its resolvent there are given by Eq. (6.4). 
In particular, the spectrum of $P$ in $U \backslash \Sigma_{P}$ is absolutely continuous. We single out this fact, stated in terms of the eigenvalues, in the following corollary.

Corollary 6.9. Let $\sigma_{p}(P)$ be the point spectrum of $P$. Then

$$
\sigma_{p}(P) \cap U \subseteq \Sigma_{P}
$$

6.1.1. The exceptional set $\boldsymbol{\Sigma}_{\boldsymbol{P}}$. Our aim is to identify the set $\Sigma_{P}$ introduced in Definition 6.6. It will turn out that (modulo one additional assumption on the smoothness of the spectral measure of $H$ ) we have equality of the sets in the last corollary. In other words, $\Sigma_{P}$ is the set of eigenvalues of $P$ embedded in $U$, and is necessarily discrete.

Let $\mu \in \Sigma_{P}$, so that by definition there exists a non-zero $\phi \in \mathcal{X}$ satisfying

$$
\phi=-V R^{ \pm}(\mu) \phi .
$$

In view of (6.6) we have $A(\mu) \phi=0$, and since the form $\langle A(\lambda) \phi, \phi\rangle$ is nonnegative we infer that the zero at $\lambda=\mu$ is a minimum. Thus formally this minimum is a second-order zero for the form. However, our smoothness assumption on the spectral measure (Definition 6.1) does not go so far as a second-order derivative. We therefore impose the following additional hypothesis on the spectral derivative near such a minimum.

REGULARITY ASSUMPTION ON SPECTRAL DENSITY. Let $K \subseteq U$ be compact and $\phi \in \mathcal{X}$ a solution to (6.7), where $\mu \in K$. Then there exist constants $C, \varepsilon>0$, depending only on $K$, so that

$$
<A(\lambda) \phi, \phi>\leq C|\lambda-\mu|^{1+\varepsilon}\|\phi\|_{\mathcal{X}}^{2}, \quad \lambda \in K .
$$

Remark 6.10. This assumption is satisfied if the operator-valued function $\lambda \rightarrow$ $A(\lambda) \in B\left(\mathcal{X}, \mathcal{X}_{H}^{*}\right)$ has a Hölder continuous (in the uniform operator topology) Fréchet derivative in a neighborhood of $\mu$. Indeed, in this case we have

$$
<A(\lambda) \phi, \phi>=<(A(\lambda)-A(\mu)) \phi, \phi>=\frac{d}{d \theta}<A(\theta) \phi, \phi>_{\theta \in[\mu, \lambda]}(\lambda-\mu) .
$$

Theorem 6.11. Let $V$ be symmetric and short-range, and assume that the condition of Lemma 6.5 is satisfied, so that $P=H+V$ is a self-adjoint operator. Assume, in addition, that the assumption above is satisfied and (6.8) holds. Then

$$
\Sigma_{P}=\sigma_{p}(P) \cap U .
$$

Furthermore, every eigenvalue is of finite multiplicity and the set of eigenvalues $\sigma_{p}(P)$ has no accumulation point in $U$.

\subsection{PERTURBATION OF THE DIRAC OPERATOR. .}

We now consider the operator

$$
H_{m}^{V}=H_{m}+V(x)
$$

where $H_{m}$ is the free Dirac operator given in (2.2) and $V(x)$ is a Hermitian $4 \times 4$ matrix that decays as $|x| \rightarrow \infty$.

We assume $m>0$. In this case the $\operatorname{spectrum~} \operatorname{spec}\left(H_{m}\right)$ has a "gap" $(-m, m)$ (see Equation (4.4) ) and our focus is on the finiteness of the eigenvalues therein. We refer to [9, 10, 21] for general discussion of the eigenvalues in the gap. A variational characterization of the eigenvalues is given in [20].

The spectral structure of $H_{m}$ was studied in Subsection 4.1. Recall that by (4.3) the domain of $H_{m}$ (as a self adjoint operator in $\mathcal{L}^{2}\left(\mathbb{R}^{3}, \mathbb{C}^{4}\right)$ ) is $\mathcal{H}^{1}\left(\mathbb{R}^{3}, \mathbb{C}^{4}\right)$. Using the 
terminology introduced in Subsection 6.1 it follows that , in view of Theorem 4.2 we can take

$$
\mathcal{X}=\mathcal{L}^{2, s}\left(\mathbb{R}^{3}, \mathbb{C}^{4}\right), \quad \mathcal{X}^{*}=\mathcal{L}^{2,-s}\left(\mathbb{R}^{3}, \mathbb{C}^{4}\right), s>\frac{1}{2} .
$$

Also, the space $\mathcal{X}_{H}^{*}$ can then be taken as the domain of (the closure of) $H_{m}$ in $\mathcal{L}^{2,-s}\left(\mathbb{R}^{3}, \mathbb{C}^{4}\right)$, so that

$$
\mathcal{X}_{H}^{*}=\mathcal{H}^{1,-s}\left(\mathbb{R}^{3}, \mathbb{C}^{4}\right) .
$$

For the selfadjointness of $H_{m}^{V}$ the following proposition suffices for our study here.

Proposition 6.12. Assume that $V$ is bounded and decays at infinity. Then the operator $H_{m}^{V}$ is self-adjoint with domain $\mathcal{H}^{1}\left(\mathbb{R}^{3}, \mathbb{C}^{4}\right) \subseteq \mathcal{L}^{2}\left(\mathbb{R}^{3}, \mathbb{C}^{4}\right)$. Its essential spectrum is $\mathbb{R} \backslash(-m, m)$.

Proof. By the coercivity inequality (4.2) it follows that $V$ is compact with respect to $H_{m}$, which establishes the claim by the general theory of self-adjoint perturbations.

Remark 6.13. Indeed, the self-adjointness of $H_{m}^{V}$ holds for a much wider class of potentials, see e.g. 2, Chapter 2], 23. As in the case of the classical Scrödinger operators, the stronger decay assumptions are needed when studying the LAP and the spectral derivative associated with the operator, as we proceed to do next.

In order to derive the Limiting Absorption Principle (LAP) of the perturbed operator $H_{m}^{V}$ from the general theory presented in Section 6.1 we need to assume that, for some constant $C>0$,

$$
|V(x)| \leq C(1+|x|)^{-(1+\varepsilon)}, \quad \varepsilon>0, x \in \mathbb{R}^{3} .
$$

It is readily verified that $V$ satisfies the conditions of Definition 6.4 so that it is short-range and symmetric.

Now let

$$
U=\mathbb{R} \backslash[-m, m] .
$$

In view of Theorem 4.2 and Theorem 6.8 we obtain the LAP for $H_{m}^{V}$ as follows.

Theorem 6.14. Assume that $V$ satisfies the decay assumption (6.12). Let $R_{m}^{V}(z)=$ $\left(H_{m}^{V}-z\right)^{-1}, \Im z \neq 0$. Then:

- For any $\frac{1}{2}<s<\frac{1+\varepsilon}{2}$ the limits

$$
R_{m}^{V, \pm}(\mu)=\lim _{\varepsilon \downarrow 0} R_{m}^{V}(\mu \pm i \varepsilon), \mu \in U \backslash \Sigma_{H_{m}}^{V},
$$

exist in the uniform operator topology of $B\left(\mathcal{L}^{2, s}\left(\mathbb{R}^{3}, \mathbb{C}^{4}\right), \mathcal{L}^{2,-s}\left(\mathbb{R}^{3}, \mathbb{C}^{4}\right)\right)$, where the (relatively) closed set $\Sigma_{H_{m}}^{V}$ is given by

$\Sigma_{H_{m}}^{V}=\left\{\lambda \in U /\right.$ There exists a non-zero $\phi_{\lambda} \in \mathcal{L}^{2, s}\left(\mathbb{R}^{3}, \mathbb{C}^{4}\right)$ such that $\left.\phi_{\lambda}=-V R_{m}^{ \pm}(\lambda) \phi_{\lambda}\right\}$.

- Assume that the decay assumption (6.12) is replaced by the stronger one

$$
|V(x)| \leq C(1+|x|)^{-(2+\varepsilon)}, \quad \varepsilon>0, x \in \mathbb{R}^{3},
$$

then for any $1<s<\frac{2+\varepsilon}{2}$ the limits in (6.13) exist for all $\mu \in \mathbb{R} \backslash \widetilde{\Sigma_{H_{m}}^{V}}$, where now

$\widetilde{\Sigma_{H_{m}}^{V}}=\left\{\lambda \in \mathbb{R} /\right.$ There exists a non-zero $\phi_{\lambda} \in \mathcal{L}^{2, s}\left(\mathbb{R}^{3}, \mathbb{C}^{4}\right)$ such that $\left.\phi_{\lambda}=-V R_{m}^{ \pm}(\lambda) \phi_{\lambda}\right\}$. 
Proof. The existence of the limits in (6.13) in $U \backslash \Sigma_{H_{m}}^{V}$ (resp. in $\mathbb{R} \backslash \widetilde{\Sigma_{H_{m}}^{V}}$ ) for the decay rate (6.12) (resp. (6.14) ) follows from the general theory (see Theorem 6.8) and the estimates implied by Theorem 4.2

Remark 6.15. Note that the operator $H_{m}^{V}$ is certainly defined on a dense subspace of $\mathcal{L}^{2,-s}\left(\mathbb{R}^{3}, \mathbb{C}^{4}\right)$. If it is closable and the graph-norm of its closure is equivalent to the norm $\mathcal{H}^{1,-s}\left(\mathbb{R}^{3}, \mathbb{C}^{4}\right)$, then the general theory (as in the case of the free operator, see Equation (6.11) implies that the limits (6.13) are actually obtained in the uniform operator topology of $B\left(\mathcal{L}^{2, s}\left(\mathbb{R}^{3}, \mathbb{C}^{4}\right), \mathcal{H}^{1,-s}\left(\mathbb{R}^{3}, \mathbb{C}^{4}\right)\right)$.

6.2.1. The discreteness and finiteness of eigenvalues. Our next goal is to identify the set $\Sigma_{H_{m}}^{V}$ with the (discrete) set of embedded eigenvalues. The abstract framework that enables us to do that was introduced in Subsubsection 6.1.1. We shall need to replace the short-range condition (6.12) by the stronger decay condition (6.14) on the potential $V$, even when dealing with spectral intervals that do not include the thresholds at $\pm m$.

Theorem 6.16. Let

$$
U=\mathbb{R} \backslash[-m, m] .
$$

Suppose that the potential $V$ satisfies the following decay condition:

$$
|V(x)| \leq C(1+|x|)^{-(2+\varepsilon)}, \quad \varepsilon>0, x \in \mathbb{R}^{3} .
$$

Let $\Sigma_{H_{m}}^{V}$ be as in Theorem 6.14. Then

$$
\Sigma_{H_{m}}^{V}=\sigma_{p}\left(H_{m}^{V}\right)=\text { the set of eigenvalues in } U \text {, }
$$

and this set is discrete, with all eigenvalues of finite multiplicity.

Proof. In view of Theorem6.11, we need to verify that the regularity condition (6.8) is satisfied under the decay assumption (6.15) .

The spectral derivative is given by Equation (4.6) (assuming $\lambda>m$ ):

$$
<A_{m}(\lambda) f, f>=\frac{\lambda}{\sqrt{\lambda^{2}-m^{2}}} \int_{|\xi|=\sqrt{\lambda^{2}-m^{2}}}\left|\left(\mathcal{G}_{m} f\right)_{+}(\xi)\right|^{2} d \Sigma_{\sqrt{\lambda^{2}-m^{2}}}
$$

Let $\frac{1}{2}<s<\frac{1+\varepsilon}{2}$.

Suppose that $\phi_{\mu} \in \mathcal{L}^{2, s}\left(\mathbb{R}^{3}, \mathbb{C}^{4}\right)$ is such that $\phi_{\mu}=-V R_{m}^{ \pm}(\mu) \phi_{\mu}, \mu>m$.

Thus $<A_{m}(\mu) \phi_{\mu}, \phi_{\mu}>=0$, so that $\left(\mathcal{G}_{m} \phi_{\mu}\right)_{+}(\xi)=0$ for all $\xi$ on the sphere $|\xi|=\sqrt{\mu^{2}-m^{2}}$. We need to show that

$$
<A_{m}(\lambda) \phi_{\mu}, \phi_{\mu}>\leq C|\lambda-\mu|^{1+\varepsilon} \text {. }
$$

We note that by Theorem 4.2 we have $R_{m}^{ \pm}(\mu) \phi_{\mu} \in \mathcal{H}^{1,-s}\left(\mathbb{R}^{3}, \mathbb{C}^{4}\right)$. Therefore (6.15) implies that $\phi_{\mu}=-V R_{m}^{ \pm}(\mu) \phi_{\mu} \in \mathcal{L}^{2,-s+2+\varepsilon}\left(\mathbb{R}^{3}, \mathbb{C}^{4}\right)$. It follows by (2.10) that $\mathcal{G}_{m} \phi_{\mu} \in \mathcal{H}^{\theta}$, where $\theta>2+\varepsilon-\frac{1+\varepsilon}{2}=\frac{3}{2}+\frac{1}{2} \varepsilon$.

In particular, the trace of $\nabla_{\xi} \mathcal{G}_{m} \phi_{\mu}(\xi)$ on the sphere $|\xi|=\sqrt{\lambda^{2}-m^{2}}$ is Hölder continuous (for $\lambda$ near $\mu$ ). From Equation (6.17) we infer that $\frac{d}{d \lambda}<A_{m}(\lambda) \phi_{\mu}, \phi_{\mu}>$ is Hölder continuous in a neighborhood of $\mu$. Since the nonnegative function $<$ $A_{m}(\lambda) \phi_{\mu}, \phi_{\mu}>$ has a minimum at $\lambda=\mu$, its derivative vanishes there, which yields (6.18) in view of the Hölder continuity. 
Finally we treat the case of the full line (and in particular the thresholds at $\lambda= \pm m$ are included). This is related to the second part of Theorem 6.14. In fact, the following theorem can be viewed as the "perturbed" version of the second part of Theorem 4.2 .

Theorem 6.17. Suppose that the potential $V$ satisfies the following decay condition:

$$
|V(x)| \leq C(1+|x|)^{-(3+\varepsilon)}, \quad \varepsilon>0, x \in \mathbb{R}^{3} .
$$

Let $\widetilde{\Sigma_{H_{m}}^{V}}$ be as in Theorem 6.14. Then

$$
\widetilde{\Sigma_{H_{m}}^{V}}=\sigma_{p}\left(H_{m}^{V}\right)=\text { the set of eigenvalues in } \mathbb{R},
$$

and this set is discrete, with all eigenvalues of finite multiplicity.

Proof. We follow the line of proof of Theorem 6.16, subject to modifications needed due to the fact that we are now looking at a full neighborhood of the thresholds.

Without loss of generality let us consider a neighborhood of $\lambda=m$.

Let $1<s<\frac{2+\varepsilon}{2}$.

According to the second part of Theorem 6.14 the limits (6.13) exist in $\mathbb{R} \backslash \Sigma_{H_{m}}^{V}$.

Suppose that $\phi_{\mu} \in \mathcal{L}^{2, s}\left(\mathbb{R}^{3}, \mathbb{C}^{4}\right)$ is such that $\phi_{\mu}=-V R_{m}^{ \pm}(\mu) \phi_{\mu}$, where $\mu \in$ $(m-\eta, m+\eta)$, for some small $\eta>0$.

Thus $<A_{m}(\mu) \phi_{\mu}, \phi_{\mu}>=0$. For $\mu \geq m$ this means that $\left(\mathcal{G}_{m} \phi_{\mu}\right)_{+}(\xi)=0$ for all $\xi$ on the sphere $|\xi|=\sqrt{\mu^{2}-m^{2}}$.

In view of Theorem 6.11, we need to verify that the regularity condition (6.8) is satisfied under the decay assumption (6.19).

We need to show that, for some $C>0$ depending only on $\eta$,

$$
<A_{m}(\lambda) \phi_{\mu}, \phi_{\mu}>\leq C|\lambda-\mu|^{1+\varepsilon}, \quad \lambda, \mu \in(m-\eta, m+\eta) .
$$

Remark that $\left.<A_{m}(\lambda) \phi_{\mu}, \phi_{\mu}\right\rangle=0$ for $\lambda \in[-m, m]$.

We note that by Theorem 4.2 we have $R_{m}^{ \pm}(\mu) \phi_{\mu} \in \mathcal{H}^{1,-s}\left(\mathbb{R}^{3}, \mathbb{C}^{4}\right)$. Therefore (6.19) implies that $\phi_{\mu}=-V R_{m}^{ \pm}(\mu) \phi_{\mu} \in \mathcal{L}^{2,-s+3+\varepsilon}\left(\mathbb{R}^{3}, \mathbb{C}^{4}\right)$. It follows by (2.10) that $\mathcal{G}_{m} \phi_{\mu} \in \mathcal{H}^{\theta}$, where $\theta>3+\varepsilon-\frac{2+\varepsilon}{2}=2+\frac{1}{2} \varepsilon$.

In particular, the trace of $\nabla_{\xi} \mathcal{G}_{m} \phi_{\mu}(\xi)$ on the sphere $|\xi|=\sqrt{\lambda^{2}-m^{2}}$ can be estimated (for $\lambda$ near $\mu$ ). In view of the trace Lemma 3.1 and Equation (6.17) we infer that, for $\lambda \in[m, m+\eta)$, the derivative $\frac{d}{d \lambda}<A_{m}(\lambda) \phi_{\mu}, \phi_{\mu}>$ is Hölder continuous and

$$
\left|\frac{d}{d \lambda}<A_{m}(\lambda) \phi_{\mu}, \phi_{\mu}>\right| \leq C\left(\sqrt{\lambda^{2}-m^{2}}\right)^{2+\varepsilon-1-1}=C\left(\lambda^{2}-m^{2}\right)^{\frac{\varepsilon}{2}} .
$$

Now

$$
<A_{m}(\mu) \phi_{\mu}, \phi_{\mu}>=\frac{d}{d \lambda}<A_{m}(\lambda) \phi_{\mu}, \phi_{\mu}>\left.\right|_{\lambda=\mu}=0,
$$

so that (6.21) follows readily, as in the conclusion of the proof of Theorem 6.16

\section{Corollary 6.18.}

$$
H_{m}^{V}=H_{m}+V(x)
$$

where $H_{m}$ is the free Dirac operator given in (2.2) and the potential $V(x)$ is a Hermitian $4 \times 4$ matrix satisfying the decay condition (6.19). 
Then $H_{m}^{V}$ has at most a discrete sequence of eigenvalues in $\mathbb{R}$. In particular, it has at most a finite number of eigenvalues in the "gap" $(-m, m)$.

Remark 6.19. Using a stronger decay hypothesis (typically $|V(x)| \leq C(1+|x|)^{-5-\varepsilon}$ ) it was shown in [12, Proposition 2.3] that there are no eigenvalues of $H_{m}^{V}$ near the thresholds at $\pm m$.

More generally, Cojuhari [16] established the finiteness of the number of eigenvalues in the gap under the weaker condition (6.15) (which in our study served only for the discreteness of embedded eigenvalues).

Remark 6.20. For a class of potentials, including the Coulomb potential, it was shown [43, Theorem 4.21] that there are actually no embedded eigenvalues in the essential spectrum $\mathbb{R} \backslash(-m, m)$.

On the other hand, for the special potential $V(x)=\gamma /|x|,|\gamma| \leq \frac{\sqrt{3}}{2}$, it is known ( [2, Section 3.1], 21]) that there is an infinite sequence of eigenvalues in the gap $(-m, m)$.

\section{GLOBAL SPACETIME ESTIMATES -FREE DIRAC and MAXWELL OPERATORS}

As in the case of the spectral study above, we deal first with spacetime estimates for the free ("unperturbed") Dirac and Maxwell operators. Our treatment here follows the method used for the case of generalized wave equations in [4, 5].

\subsection{SPACETIME ESTIMATES FOR THE FREE DIRAC OPERATOR.}

The free Dirac operator was defined in (2.2). We consider the unitary group associated with it

$$
i u_{t}=H_{m} u, \quad t \in \mathbb{R},
$$

subject to the initial condition

$$
u(x, 0)=u_{0}(x), \quad x \in \mathbb{R}^{3},
$$

where $u, u_{0}$ are $\mathbb{C}^{4}$-valued functions.

We refer to [19] for Strichartz estimates for the Dirac operator with magnetic potentials. Here we formulate a global spacetime estimate in the weighted $-L^{2}$ framework.

Theorem 7.1. Let $m>0, s>1$, and let $u(x, t)$ be the solution to (7.1)-(7.2). There exists a constant $C=C_{s, m}>0$, such that

$$
\int_{\mathbb{R}} \int_{\mathbb{R}^{3}}\left(1+|x|^{2}\right)^{-s}|u(x, t)|^{2} d x d t \leq C\left\|u_{0}\right\|_{0}^{2} .
$$

Proof. The solution $u(x, t)$ can be represented as

$$
u=\exp \left(-i t H_{m}\right) u_{0} .
$$

In the proof we find it clearer to use the separate notations $(\cdot, \cdot),[\cdot, \cdot]$ for the scalar products in $\mathcal{L}^{2}\left(\mathbb{R}^{3}, \mathbb{C}^{4}\right), \mathcal{L}^{2}\left(\mathbb{R}^{4}, \mathbb{C}^{4}\right)$, respectively.

We denote by

$$
\tilde{v}(x, \tau)=(2 \pi)^{-\frac{1}{2}} \int_{\mathbb{R}} v(x, t) e^{-i t \tau} d t
$$


the partial Fourier transform with respect to $t$.

The spectral derivative $A_{m}$ was defined in equation (4.7).

To estimate $u(x, t)$ we use a duality argument. Take $w(x, t) \in C_{0}^{\infty}\left(\mathbb{R}^{4}, \mathbb{C}^{4}\right)$. Then,

$$
\begin{aligned}
& {[u, w]=\int_{-\infty}^{\infty} d t \int_{\mathbb{R}^{3}}<\left(e^{-i t H_{m}} u_{0}\right)(x, t), w(x, t)>_{\mathbb{C}^{4}} d x} \\
& =\int_{-\infty}^{\infty}<A_{m}(\lambda) u_{0}, \int_{-\infty}^{\infty} e^{-i t \lambda} w(\cdot, t) d t>d \lambda, \\
& =(2 \pi)^{1 / 2} \int_{-\infty}^{\infty}<A_{m}(\lambda) u_{0}, \tilde{w}(\cdot, \lambda)>d \lambda .
\end{aligned}
$$

where in the last two terms $<,>$ is the $\left(\mathcal{L}^{2,-s}\left(\mathbb{R}^{3}, \mathbb{C}^{4}\right), \mathcal{L}^{2, s}\left(\mathbb{R}^{3}, \mathbb{C}^{4}\right)\right)$ pairing.

We note that by the spectral theorem

$$
\int_{-\infty}^{\infty}<A_{m}(\lambda) f, f>d \lambda=\|f\|_{0}^{2} .
$$

The Cauchy-Schwarz inequality yields

$$
\begin{gathered}
\leq(2 \pi)^{1 / 2}\left(\int_{-\infty}^{\infty}<A_{m}(\lambda) u_{0}, u_{0}>d \lambda\right)^{1 / 2} \cdot\left(\int_{-\infty}^{\infty}<A_{m}(\lambda) \tilde{w}(\cdot, \lambda), \tilde{w}(\cdot, \lambda)>d \lambda\right)^{1 / 2} \\
=(2 \pi)^{1 / 2}\left\|u_{0}\right\|_{2} \cdot\left(\int_{-\infty}^{\infty}<A_{m}(\lambda) \tilde{w}(\cdot, \lambda), \tilde{w}(\cdot, \lambda)>d \lambda\right)^{1 / 2} .
\end{gathered}
$$

and recalling (4.7) we obtain

$$
\begin{gathered}
|[u, w]| \\
\leq(2 \pi)^{1 / 2} C\left\|u_{0}\right\|_{0} \cdot\left(\int_{|\lambda|>m} \min \left(\frac{|\lambda|}{\sqrt{\lambda^{2}-m^{2}}},|\lambda|\left(\lambda^{2}-m^{2}\right)^{s-1}\right) \cdot\|\tilde{w}(\cdot, \lambda)\|_{0, s}^{2} d \lambda\right)^{\frac{1}{2}} .
\end{gathered}
$$

By the Plancherel theorem, using $s>1$,

$$
|[u, w]| \leq(2 \pi)^{1 / 2} C\left\|u_{0}\right\|_{0}\left(\int_{\mathbb{R}}\|w(\cdot, t)\|_{0, s}^{2} d t\right)^{1 / 2} .
$$

Let $f(x, t) \in C_{0}^{\infty}\left(\mathbb{R}^{4}, \mathbb{C}^{4}\right)$, and take $w(x, t)=\left(1+|x|^{2}\right)^{-\frac{s}{2}} f(x, t)$, so that

$$
\int_{\mathbb{R}}\|w(\cdot, t)\|_{0, s}^{2} d t=\|f\|_{0}^{2}
$$

We infer that

$$
\left|\left[\left(1+|x|^{2}\right)^{-\frac{s}{2}} u, f\right]\right| \leq(2 \pi)^{1 / 2} C \cdot\left\|u_{0}\right\|_{0} \cdot\|f\|_{0},
$$

which concludes the proof of the theorem. 
Remark 7.2. Note that we need only (for the unperturbed case) $s>1$, including the propagation near the threshold. This is to be compared with Theorem 1.1 and Proposition 2.1 in [12], where $s>\frac{5}{2}$, is assumed (including a potential).

\subsection{SPACETIME ESTIMATES FOR THE FREE MAXWELL OPER-} ATOR. .

The free Maxwell operator $L_{\text {maxwell }}$ was introduced in (2.14).

We consider the unitary group associated with the operator,

$$
i u_{t}=L_{\text {maxwell }} u, \quad t \in \mathbb{R},
$$

subject to the initial condition

$$
u(x, 0)=u_{0}(x), \quad x \in \mathbb{R}^{3},
$$

where $u, u_{0}$ are $\mathbb{C}^{6}$-valued functions.

We further assume that

$$
u_{0} \in(I-\mathcal{P}) \mathcal{L}^{2}\left(\mathbb{R}^{3}, \mathbb{C}^{6}\right),
$$

where $\mathcal{P}$ is the orthogonal projection on $\operatorname{ker}\left(L_{\text {maxwell }}\right)$ (see Equation (4.25)).

In view of Remark 2.6 the orthogonality condition means that if $u_{0}(x)=\left(\begin{array}{l}E_{0}(x) \\ B_{0}(x)\end{array}\right)$ (with values in $\mathbb{C}^{6}$ ) then Fourier transform $\left(\frac{\widehat{E_{0}}(\xi)}{\widehat{B_{0}}(\xi)}\right) \in \operatorname{span}\left\{\Upsilon_{ \pm}\right\}$, for every $0 \neq \xi \in$ $\mathbb{R}^{3}$. In particular

$$
<\widehat{E_{0}}(\xi), \xi>_{\mathbb{C}^{3}}=0, \quad<\widehat{B_{0}}(\xi), \xi>_{\mathbb{C}^{3}}=0, \quad \xi \in \mathbb{R}^{3} \backslash\{0\},
$$

so that $E_{0}$ (resp. $\left.B_{0}\right)$ is a "TE-mode" (resp. "TM-mode").

Theorem 7.3. Let $s>\frac{1}{2}$, and let $u(x, t)$ be the solution to (7.4) -(7.5)-(7.6). There exists a constant $C=C_{s}>0$, such that

$$
\int_{\mathbb{R}} \int_{\mathbb{R}^{3}}\left(1+|x|^{2}\right)^{-s}|u(x, t)|^{2} d x d t \leq C\left\|u_{0}\right\|_{0}^{2} .
$$

Proof. The solution $u(x, t)$ can be represented as

$$
u=\exp \left(-i t L_{\text {maxwell }}\right) u_{0} .
$$

As in the proof of Theorem 7.1, we find it clearer to use the separate notations $(\cdot, \cdot),[\cdot, \cdot]$ for the scalar products in $\mathcal{L}^{2}\left(\mathbb{R}^{3}, \mathbb{C}^{6}\right), \mathcal{L}^{2}\left(\mathbb{R}^{4}, \mathbb{C}^{6}\right)$, respectively.

We denote by

$$
\tilde{v}(x, \tau)=(2 \pi)^{-\frac{1}{2}} \int_{\mathbb{R}} v(x, t) e^{-i t \tau} d t
$$

the partial Fourier transform with respect to $t$.

The spectral derivative $\widetilde{A}$ was defined in equation (4.28). 
To estimate $u(x, t)$ we use a duality argument. Take $\left.w(x, t) \in C_{0}^{\infty}\left(\mathbb{R}^{4}, \mathbb{C}^{6}\right)\right)$. Then,

$$
\begin{aligned}
& {[u, w]=\int_{-\infty}^{\infty} d t \int_{\mathbb{R}^{3}}<\left(e^{-i t L_{\text {maxwell }}} u_{0}\right)(x, t), w(x, t)>_{\mathbb{C}^{6}} d x} \\
& =\int_{-\infty}^{\infty}<\widetilde{A}(\lambda) u_{0}, \int_{-\infty}^{\infty} e^{-i t \lambda} w(\cdot, t) d t>d \lambda \\
& =(2 \pi)^{1 / 2} \int_{-\infty}^{\infty}<\widetilde{A}(\lambda) u_{0}, \tilde{w}(\cdot, \lambda)>d \lambda .
\end{aligned}
$$

We note that by the spectral theorem

$$
\int_{-\infty}^{\infty}<\widetilde{A}(\lambda) f, f>d \lambda=\|f\|_{0}^{2} .
$$

The Cauchy-Schwarz inequality yields

$$
\begin{gathered}
\leq(2 \pi)^{1 / 2}\left(\int_{-\infty}^{\infty}<\tilde{A}(\lambda) u_{0}, u_{0}>d \lambda\right)^{1 / 2} \cdot\left(\int_{-\infty}^{\infty}<\widetilde{A}(\lambda) \tilde{w}(\cdot, \lambda), \tilde{w}(\cdot, \lambda)>d \lambda\right)^{1 / 2} \\
=(2 \pi)^{1 / 2}\left\|u_{0}\right\|_{2} \cdot\left(\int_{-\infty}^{\infty}<\widetilde{A}(\lambda) \tilde{w}(\cdot, \lambda), \tilde{w}(\cdot, \lambda)>d \lambda\right)^{1 / 2}
\end{gathered}
$$

and recalling (4.28) we obtain

$$
\begin{aligned}
& |[u, w]| \\
& \leq(2 \pi)^{1 / 2} C\left\|u_{0}\right\|_{0} \cdot\left(\int_{-\infty}^{\infty} \min \left(1,|\lambda|^{2 s-1}\right) \cdot\|\tilde{w}(\cdot, \lambda)\|_{0, s}^{2} d \lambda\right)^{\frac{1}{2}} .
\end{aligned}
$$

By the Plancherel theorem, using $s>\frac{1}{2}$,

$$
|[u, w]| \leq(2 \pi)^{1 / 2} C\left\|u_{0}\right\|_{0}\left(\int_{\mathbb{R}}\|w(\cdot, t)\|_{0, s}^{2} d t\right)^{1 / 2} .
$$

Let $f(x, t) \in C_{0}^{\infty}\left(\mathbb{R}^{4}, \mathbb{C}^{6}\right)$, and take $w(x, t)=\left(1+|x|^{2}\right)^{-\frac{s}{2}} f(x, t)$, so that

$$
\int_{\mathbb{R}}\|w(\cdot, t)\|_{0, s}^{2} d t=\|f\|_{0}^{2}
$$

We infer that

$$
\left|\left[\left(1+|x|^{2}\right)^{-\frac{s}{2}} u, f\right]\right| \leq(2 \pi)^{1 / 2} C \cdot\left\|u_{0}\right\|_{0} \cdot\|f\|_{0},
$$

which concludes the proof of the theorem. 


\section{GLOBAL SPACETIME ESTIMATES -STRONGLY PROPAGATIVE HOMOGENEOUS SYSTEMS}

We now address the spacetime decay estimates for homogeneous strongly prop-

agative operators $L_{0}=\sum_{j=1}^{n} M_{j}^{0} D_{j}$ (see (1.2) $)$, for which the LAP was stated in Theorem5.13. Recall that both the Dirac (zero mass) and Maxwell systems belong to this class, but the singular set $Z$ (defined in (5.2)) is empty in both cases.

We consider the associated unitary group

$$
i u_{t}=L_{0, h o m} u, \quad t \in \mathbb{R},
$$

subject to the initial condition

$$
u(x, 0)=u_{0}(x), \quad x \in \mathbb{R}^{n},
$$

where $u, u_{0}$ are $\mathbb{C}^{K}$-valued functions.

We further assume that the initial function is orthogonal to the "stationary waves" of the system, namely,

$$
u_{0} \in(I-\mathcal{P}) \mathcal{L}^{2}\left(\mathbb{R}^{n}, \mathbb{C}^{K}\right),
$$

where $\mathcal{P}$ is the orthogonal projection on $\operatorname{ker}\left(L_{0, \text { hom }}\right)$.

Clearly the solution $u(x, t)=e^{-i t L_{0, h o m}} u_{0}$ satisfies $u(\cdot, t) \in(I-\mathcal{P}) \mathcal{L}^{2}\left(\mathbb{R}^{n}, \mathbb{C}^{K}\right), t \in$ $\mathbb{R}$.

It is represented by the Fourier integral operator

$$
u(x, t)=(2 \pi)^{-\frac{n}{2}} \int_{\mathbb{R}^{n}} e^{-i t \sum_{j=1}^{n} M_{j}^{0} \xi_{j}} e^{-i<\xi, x>} \widehat{u_{0}}(\xi) d \xi .
$$

However, this explicit expression does not easily lend itself to asymptotic analysis by classical methods of geometric optics; the non-commutativity of the matrices $\left\{M_{j}^{0}\right\}_{j=1}^{n}$ requires a very detailed study of the algebraic structure of the eigenvalue manifolds and their intersections. Hence various restrictive hypotheses need to be imposed, even in the case of constant coefficients.

There is extensive literature concerning the (large-time) asymptotic behavior of solutions of such systems and their perturbations, using primarily the geometric optics approach. It is beyond the scope of the present paper to present an exhaustive account of these works, and we refer to [36] and references therein. In particular it is assumed there that the system is uniformly propagative [36, Assumption (1.3)]. The rate of decay in time of the $L^{2}$ norm of scattered solutions in balls is shown [36, Theorem 1] to be $O\left((\log t)^{-1}\right)$. Confining to the constant coefficient case, the global estimate (8.13) obtained below yields a faster rate of decay (in integral sense).

The presence of the singular set $Z$ makes our spacetime estimate somewhat more delicate. Recall Definition [5.7 of the closed subspace $\Upsilon_{Z}^{s} \subseteq \mathcal{L}^{2, s}\left(\mathbb{R}^{n}, \mathbb{C}^{K}\right)$, and note also Remark [5.10. We now define the closed subspace $\Upsilon_{Z} \subseteq \mathcal{L}^{2}\left(\mathbb{R}^{n}, \mathbb{C}^{K}\right)$ as the inverse Fourier transform of $(I-\Delta)^{\frac{s}{2}} \widehat{\Upsilon_{Z}^{s}}$, namely

$$
\Upsilon_{Z}=\left(1+|x|^{2}\right)^{\frac{s}{2}} \Upsilon_{Z}^{s}=\left\{g / g(x)=\left(1+|x|^{2}\right)^{\frac{s}{2}} h(x) \text { for some } h \in \Upsilon_{Z}^{s}\right\} .
$$

Let $\mathcal{E}: \mathcal{L}^{2}\left(\mathbb{R}^{n}, \mathbb{C}^{K}\right) \hookrightarrow \Upsilon_{Z}$ be the orthogonal projection unto this subspace.

Theorem 8.1. Assume that $L_{0, \text { hom }}$ is strongly propagative. Let $s>\frac{1}{2}$, and let $u(x, t)$ be the solution to (8.1) $-(8.2)-(8.3)$. 
Define $u_{Z}(x, t), t \in \mathbb{R}$, by

$$
u_{Z}(x, t)=\mathcal{E}\left[\left(1+|x|^{2}\right)^{-\frac{s}{2}} u(x, t)\right] .
$$

Then there exists a constant $C=C_{s, n}>0$, such that

$$
\int_{\mathbb{R}} \int_{\mathbb{R}^{n}}\left|u_{Z}(x, t)\right|^{2} d x d t \leq C\left\|u_{0}\right\|_{0}^{2} .
$$

Remark 8.2. Note that $u_{Z}(x, t) \in \Upsilon_{Z}$ implies $\left(1+|x|^{2}\right)^{-\frac{s}{2}} u_{Z}(x, t) \in \Upsilon_{Z}^{s}$ for every $t \in \mathbb{R}$. Fix $t=t_{0} \in \mathbb{R}$. In light of Definition [5.7, given $\eta>0$ there exists $\psi_{\eta} \in \mathcal{L}^{2, s}\left(\mathbb{R}^{n}, \mathbb{C}^{K}\right)$ so that $\widehat{\psi_{\eta}} \in C_{0}^{\infty}\left(\mathbb{R}^{n} \backslash \bar{Z}, \mathbb{C}^{K}\right)$ and

$$
\int_{\mathbb{R}^{n}}\left(1+|x|^{2}\right)^{s}\left|\left(1+|x|^{2}\right)^{-\frac{s}{2}} u_{Z}\left(x, t_{0}\right)-\psi_{\eta}(x)\right|^{2} d x \leq \eta^{2} .
$$

Thus

$$
\int_{\mathbb{R}^{n}}\left|u_{Z}\left(x, t_{0}\right)-\left(1+|x|^{2}\right)^{\frac{s}{2}} \psi_{\eta}(x)\right|^{2} d x \leq \eta^{2},
$$

and by the Plancherel theorem

$$
\int_{\mathbb{R}^{n}}\left|\widehat{u_{Z}}\left(\xi, t_{0}\right)-(I-\Delta)^{\frac{s}{2}} \widehat{\psi_{\eta}}(\xi)\right|^{2} d \xi \leq \eta^{2} .
$$

The function $u_{Z}\left(x, t_{0}\right)$ is therefore the part of $u\left(x, t_{0}\right)$ whose Fourier transform is in the closure (in $\mathcal{L}^{2}\left(\mathbb{R}^{n}, \mathbb{C}^{K}\right)$ ) of the range of $(I-\Delta)^{\frac{s}{2}}$ acting on smooth functions "supported away" from the singular set $\bar{Z}$.

Proof of theorem: By density (in $\mathcal{L}^{2}\left(\mathbb{R}^{n}, \mathbb{C}^{K}\right)$ ) we may assume

$$
\widehat{u_{0}}(\xi) \in C_{0}^{\infty}\left(\mathbb{R}^{n} \backslash \bar{Z}, \mathbb{C}^{K}\right) .
$$

The solution $u(x, t)$ can be represented as

$$
u=\exp \left(-i t L_{0, h o m}\right) u_{0} .
$$

In particular, $\widehat{u}(\cdot, t) \in C_{0}^{\infty}\left(\mathbb{R}^{n} \backslash \bar{Z}, \mathbb{C}^{K}\right)$ for all $t \in \mathbb{R}$.

As in the proofs of Theorems 7.1 and 7.3 , we find it clearer to use the separate notations $(\cdot, \cdot),[\cdot, \cdot]$ for the scalar products in $\mathcal{L}^{2}\left(\mathbb{R}^{n}, \mathbb{C}^{K}\right), \mathcal{L}^{2}\left(\mathbb{R}^{n+1}, \mathbb{C}^{K}\right)$, respectively.

We denote by

$$
\tilde{\gamma}(x, \tau)=(2 \pi)^{-\frac{1}{2}} \int_{\mathbb{R}} \gamma(x, t) e^{-i t \tau} d t
$$

the partial Fourier transform with respect to $t$.

The spectral derivative $\widetilde{A_{0, h o m}}(\lambda) \in B\left(\Upsilon_{Z}^{s}, \mathcal{L}^{2,-s}\left(\mathbb{R}^{n}, \mathbb{C}^{K}\right)\right), s>\frac{1}{2}$, was defined in Equation (5.23).

To estimate $u(x, t)$ we use a duality argument, as in the cases of the Dirac and Maxwell systems. Take $w(x, t) \in C^{\infty}\left(\mathbb{R}^{n+1}, \mathbb{C}^{K}\right)$, so that

$$
\widehat{w}(\xi, t) \in C_{0}^{\infty}\left(\left(\mathbb{R}^{n} \backslash \bar{Z}\right) \times \mathbb{R}, \mathbb{C}^{K}\right) .
$$


Then,

$$
\begin{aligned}
& {[u, w]=\int_{-\infty}^{\infty} d t \int_{\mathbb{R}^{3}}<\left(e^{-i t L_{0, h o m}} u_{0}\right)(x, t), w(x, t)>_{\mathbb{C}^{K}} d x} \\
& =\int_{-\infty}^{\infty}<\widetilde{A_{0, h o m}}(\lambda) u_{0}, \int_{-\infty}^{\infty} e^{-i t \lambda} w(\cdot, t) d t>d \lambda \\
& =(2 \pi)^{1 / 2} \int_{-\infty}^{\infty}<\widetilde{A_{0, h o m}}(\lambda) u_{0}, \tilde{w}(\cdot, \lambda)>d \lambda .
\end{aligned}
$$

We note that by the spectral theorem

$$
\int_{-\infty}^{\infty}<\widetilde{A_{0, h o m}}(\lambda) u_{0}, u_{0}>d \lambda=\left\|u_{0}\right\|_{0}^{2}
$$

The Cauchy-Schwarz inequality yields

$$
\begin{array}{r}
|[u, w]| \\
\leq(2 \pi)^{1 / 2}\left(\int_{-\infty}^{\infty}<\widetilde{A_{0, h o m}}(\lambda) u_{0}, u_{0}>d \lambda\right)^{1 / 2} \cdot\left(\int_{-\infty}^{\infty}<\widetilde{A_{0, h o m}}(\lambda) \tilde{w}(\cdot, \lambda), \tilde{w}(\cdot, \lambda)>d \lambda\right)^{1 / 2} \\
=(2 \pi)^{1 / 2}\left\|u_{0}\right\|_{2} \cdot\left(\int_{-\infty}^{\infty}<\widetilde{A_{0, \text { hom }}}(\lambda) \tilde{w}(\cdot, \lambda), \tilde{w}(\cdot, \lambda)>d \lambda\right)^{1 / 2} .
\end{array}
$$

and recalling (5.24) and the fact that the norm of $\Upsilon_{Z}^{s}$ is the $\mathcal{L}^{2, s}$ norm, it follows that

$$
|[u, w]| \leq(2 \pi)^{1 / 2} C\left\|u_{0}\right\|_{0} \cdot \int_{-\infty}^{\infty}\|\tilde{w}(\cdot, \lambda)\|_{0, s}^{2} d \lambda
$$

Invoking the Plancherel theorem, this estimate leads to

$$
|[u, w]| \leq(2 \pi)^{1 / 2} C\left\|u_{0}\right\|_{0}\left(\int_{\mathbb{R}}\|w(\cdot, t)\|_{0, s}^{2} d t\right)^{1 / 2} .
$$

The estimate (8.11) was obtained under the condition (8.8), and by closure it holds for all $w(x, t) \in \mathcal{L}^{2}\left(\mathbb{R}_{t}, \Upsilon_{Z}^{s}\right)$.

Now note that $v(x, t)=\left(1+|x|^{2}\right)^{\frac{s}{2}} w(x, t) \in \mathcal{L}^{2}\left(\mathbb{R}_{t}, \Upsilon_{Z}\right)$.

The estimate (8.11) can be rewritten as

$$
\left|\left[\left(1+|x|^{2}\right)^{-\frac{s}{2}} u, v\right]\right| \leq(2 \pi)^{1 / 2} C\left\|u_{0}\right\|_{0}\left(\int_{\mathbb{R}}\|v(\cdot, t)\|_{0}^{2} d t\right)^{1 / 2}, \quad \forall v(\cdot, t) \in \mathcal{L}^{2}\left(\mathbb{R}_{t}, \Upsilon_{Z}\right),
$$

which clearly entails (8.6). 
Corollary 8.3. Assume that $L_{0, h o m}$ is uniformly propagative (Definition 5.3). Let $s>\frac{1}{2}$, and let $u(x, t)$ be the solution to (8.1)-(8.2)-(8.3).

Then there exists a constant $C=C_{s, n}>0$, such that

$$
\int_{\mathbb{R}} \int_{\mathbb{R}^{n}}\left(1+|x|^{2}\right)^{-s}|u(x, t)|^{2} d x d t \leq C\left\|u_{0}\right\|_{0}^{2}
$$

Proof. In this case by definition $Z=\emptyset$ so that $u_{Z}(x, t)=\left(1+|x|^{2}\right)^{-\frac{s}{2}} u(x, t)$ in Theorem 8.1 .

Remark 8.4. Note that if Conjecture[5.11 is shown to be true, then $\Upsilon_{Z}=\mathcal{L}^{2}\left(\mathbb{R}^{n}, \mathbb{C}^{K}\right)$ and $u_{Z}=\left(1+|x|^{2}\right)^{-\frac{s}{2}} u(x, t)$ for any strongly propagative system.

The result in Theorem 8.1 involved the "projected" function $u_{Z}$ because we let the initial function $u_{0}$ be any function in $\mathcal{L}^{2}\left(\mathbb{R}^{n}, \mathbb{C}^{K}\right)$. However, if we restrict the support of $u_{0}$ away from $\bar{Z}$ we can get an improved estimate, but with a constant that depends on the support of $u_{0}$ as follows.

Theorem 8.5. Assume that $L_{0, h o m}$ is strongly propagative. Let $s>\frac{1}{2}$, and let $u(x, t)$ be the solution to (8.1)-(8.2) $-(8.3)$. Let $\mathfrak{K} \Subset \mathbb{R}^{n} \backslash \bar{Z}$ be a compact set and assume further that $\operatorname{supp}\left[\widehat{u}_{0}\right] \subseteq \mathfrak{K}$.

Then there exists a constant $C=C_{s, n, \mathfrak{K}}>0$ such that

$$
\int_{\mathbb{R}} \int_{\mathbb{R}^{n}}\left(1+|x|^{2}\right)^{-s}|u(x, t)|^{2} d x d t \leq C\left\|u_{0}\right\|_{0}^{2}
$$

Proof. We repeat the proof of Theorem 8.1. However instead of (8.8) we take any $\widehat{w}(\xi, t) \in C_{0}^{\infty}\left(\left(\mathbb{R}^{n+1}, \mathbb{C}^{K}\right)\right.$. Now let $\chi(\xi) \in C_{0}^{\infty}\left(\mathbb{R}^{n} \backslash \bar{Z}\right)$ so that $\chi \equiv 1$ on a compact neighborhood of $\mathfrak{K}$. Define $\widehat{w_{1}}(\xi, t)=\chi(\xi) \widehat{w}(\xi, t)$ and let $w_{1}(x, t)$ be the inverse Fourier transform. Clearly $w_{1}(x, t) \in \mathcal{L}^{2}\left(\mathbb{R}_{t}, \Upsilon_{Z}^{s}\right)$ and with the notation of (8.9) we have

$$
\left[u, w_{1}\right]=[u, w] .
$$

The proof now proceeds as before and is completed by noting that with a constant $C=C_{s, n, \mathfrak{K}}>0$

$$
\left\|w_{1}(\cdot, t)\right\|_{0, s} \leq C\|w(\cdot, t)\|_{0, s}, \quad t \in \mathbb{R} .
$$

\section{REFERENCES}

[1] S. Agmon and L. Hörmander, Asymptotic properties of solutions of differential equations with simple characteristics, Jour. d'Analyse Math. 30 (1976), 1-38.

[2] A. A. Balinsky and W.D. Evans, "Spectral Analysis of Relativistic Operators", Imperial College Press, 2011.

[3] E. Balslev and B. Helffer, Limiting absorption principle and resonances for the Dirac operator, Adv. Appl. Math. 13 (1992), 186-215.

[4] M. Ben-Artzi, Eigenfunction expansions and spacetime estimates for generators in divergence-form, Reviews in Math. Phys. 22 (2010), 1209-1240.

[5] M. Ben-Artzi, Smooth spectral calculus, In: M. Demuth, B.-W. Schulze and I. Witt (Eds.) "Partial Differential Equations and Spectral Theory", pp. 119-182 (vol. 211 in the series "Operator Theory: Advances and Applications"). Springer Basel, 2010.

[6] M. Ben-Artzi and A. Devinatz, Resolvent estimates for a sum of tensor products with applications to the spectral theory of differential operators, Jour. d'Analyse Math. 43 (1983/4), $215-250$. 
[7] M.Ben-Artzi and A. Devinatz, "The limiting absorption principle for partial differential operators", Memoirs of the AMS 364 (1987).

[8] M. Ben-Artzi and J. Nemirovsky, Remarks on relativistic Schrödinger operators and their extensions, Ann. Inst. H. Poincaré-Phys. Théorique 67 (1997), 29-39.

[9] A. Berthier and V. Georgescu, On the point spectrum of Dirac operators, J. Funct. Anal. 71 (1987), 309-338.

[10] M. Sh. Birman and A. Laptev, Discrete spectrum of the perturbed Dirac operator, Ark. Mat. 32 (1994), 13-32 .

[11] M. Sh. Birman and M. Z. Solomyak, $L^{2}$ theory of the Maxwell operator in arbitrary domains, Russian Math. Surveys 42 (1987), 75-96 (Original paper: Uspekhi Math. Nauk 42 (1987), 61-76).

[12] N. Boussaid, Stable directions for small nonlinear Dirac standing waves, Comm. Math. Phys.268 (2006), 757-817.

[13] N. Boussaid and S. Golénia, Limiting absorption principle for some long range perturbations of Dirac systems at threshold energies, Comm. Math. Phys.299 (2010), 677-708.

[14] A. Boutet de Monvel-Berthier, D. Manda and R. Purice, Limiting absorption principle for the Dirac operator, Annal. l'I.H.P., section A, 58 (1993), 413-431.

[15] A. Carey, F. Gesztesy, J. Kaad, G. Levitina, R.Nichols, D. Potapov and F. Sukochev, On the global limiting absorption principle for massless Dirac operators, Ann. Henri Poincaré, 19 (2018), 1993-2019.

[16] P. A. Cojuhari, On the finiteness of the discrete spectrum of the Dirac operator, Reports Math. Phys.57 (2006), 333-341.

[17] R. Courant and D. Hilbert, "Methods of Mathematical Physics, Vol. 2 ", Interscience Publishers, 1962

[18] P. D'Ancona and L. Fanelli, Decay estimates for the wave and Dirac equations with a magnetic potential, Comm. Pure Appl. Math. 60 (2007), 357-392.

[19] P. D'Ancona and L. Fanelli, Strichartz and smoothing for dispersive equations with magnetic potentials, Comm. Partial Diff. Eqs. 33 (2008), 1082-1112.

[20] J. Dolbeault, M. J. Esteban and E. Séré, Variational characterization for eigenvalues of Dirac operators , Calc. Var. Partial Diff. Eqs. 10 (2000), 321-347.

[21] J. Dolbeault, M. J. Esteban and E. Séré, On the eigenvalues of operators with gaps. Application to Dirac operators, J. Funct. Anal.174 (2000), 208-226.

[22] M. B. Erdoğan, M. Goldberg and W.R. Green, Limiting absorption principle and Strichartz estimates for Dirac operators in two and higher dimensions, arXiv:1706.05257v1 (2017).

[23] M. J. Esteban and M. Loss, Self-adjointness for Dirac operators via Hardy-Dirac inequalities, J. Math. Phys.48 (2007), 1121071-1121078.

[24] L. C. Evans, "Partial Differential Equations", American Mathematical Society 1997.

[25] N. Filonov and F. Klopp, Absolutely continuous spectrum for the isotropic Maxwell operator with coefficients that are periodic in some directions and decay in others, Comm. Math. Phys. 258 (2005), 75-85.

[26] L. L. Foldy and S. A. Wouthuysen, On the Dirac theory of spin 1/2 particles and its nonrelativistic limit ,Phys. Rev. 78 (1950), 29-35.

[27] L. Hörmander, "The Analysis of Linear Partial Differential Operators, vol. II", SpringerVerlag, 1983

[28] L. Hörmander and J. L. Lions, Sur la complétion par rapport à une intégrale de Dirichlet, Math.Scand.4 (1956), 259-270.

[29] A. Iftimovici and M. Măntoiu, Limiting absorption principle at critical values for the Dirac operator, Letters Math. Phys. 49 (1999), 235-243.

[30] T. Kato, "Perturbation Theory for Linear Operators", Springer-Verlag 1976.

[31] O. Liess, Curvature properties of the slowness surface of the system of crystal acoustics for cubic crystals, Osaka J. Math. 45 (2008), 173-210.

[32] V. Maz'ya, "Sobolev Spaces ", Springer-Verlag, 2011.

[33] K. Mochizuki, Spectral and scattering theory for symmetric hyperbolic systems in an exterior domain, Publ. RIMS, Kyoto Univ. 5 (1969), 219-258

[34] M. Murata, Rate of decay of local energy and wave operators for symmetric systems, J. Math. Soc. Japan 31 (1979), 451-480 .

[35] C. Pladdy, Y. Saito and T. Umeda, Resolvent estimates of the Dirac operator, Analysis 15 (1995), 123-149. 
[36] J. Rauch, Asymptotic behavior of solutions to hyperbolic partial differential equations with zero speeds, Comm. Pure Appl. Math. 31 (1978), 431-480

[37] Y. Saito and T. Umeda, The zero modes and zero resonances of massless Dirac operators, Hokkaido Math. J. 37 (2008), 363-388.

[38] Y. Saito and T. Umeda, Eigenfunctions at the threshold energies of magnetic Dirac operators, Reviews in Math. Phys. 23 (2011), 155-178.

[39] J. R. Schulenberger and C. H. Wilcox, The limiting absorption principle and spectral theory for steady state wave propagation in inhomogeneous anisotropic media, Arch. Rat. Mech. Anal. 41 (1972), 46-65.

[40] W. V. Smith, Average stability and decay properties of forced solutions of the wave propagation problems of classical physics in energy and mean norms, J. Math.Anal. Appl.143 (1989), 148-186.

[41] H. Tamura, The principle of limiting absorption for uniformly propagative systems with perturbations of long-range class, Nagoya Math.J.82 (1981), 141-174.

[42] H. Tamura, The principle of limiting absorption for propagative systems in crystal optics with perturbations of long-range class, Nagoya Math.J.84 (1981), 169-193.

[43] B. Thaller, "The Dirac Equation", Springer-Verlag, 1992.

[44] T. Umeda, Radiation conditions and resolvent estimates of relativistic Schrödinger operators , Ann. Inst. H. Poincaré-Phys. Théorique 63 (1995), 277-296.

[45] R. Weder, Spectral analysis of strongly propagative systems, J. Reine und Angewandte Math. 354 (1984), 95-122.

[46] R. Weder, Analyticity of the scattering matrix for wave propagation in crystals, J. Math. Pures Appl. 64 (1985), 121-148.

[47] C. H. Wilcox, Wave operators and asymptotic solutions of wave propagation problems of classical physics, Arch. Rat. Mech. Anal. 22 (1966), 37-78.

[48] C. H. Wilcox, Asymptotic wave functions and energy distributions in strongly propagative anisotropic media, J. Math. Pures Appl. 57 (1978), 275-321.

[49] K.Yajima, The limiting absorption principle for uniformly propagative systems, J. Fac. Sci. Univ. Tokyo 21 (1974), 119-131.

[50] O. Yamada, On the principle of limiting absorption for the Dirac operators, Publ. RIMS, Kyoto Univ. 8 (1972/73), 557-577.

[51] O. Yamada, A remark on the limiting absorption method for the Dirac operators, Proc. Japan Acad. Ser. A Math. Sci. 69 (1993), 243-246.

Matania Ben-Artzi: Institute of Mathematics, The Hebrew University, Jerusalem 91904, ISRAEL

E-mail address: mbartzi@math.huji.ac.il

Tomio Umeda: Department of Mathematical Sciences, University of Hyogo, Himeji 671-2201, JAPAN

E-mail address: umeda@sci.u-hyogo.ac.jp 